\title{
A Simplified General Circulation Model for a Baroclinic Ocean with Topography. Part I: Theory, Waves, and Wind-Driven Circulations
}

\author{
DiRK Olbers \\ Alfred Wegener Institute for Polar and Marine Research, Bremerhaven, Germany \\ CARSTEN EDEN* \\ Department of Oceanography, Dalhousie University, Halifax, Canada
}

(Manuscript received 28 February 2002, in final form 22 April 2003)

\begin{abstract}
A new type of ocean general circulation model with simplified physics is described and tested for various simple wind-driven circulation problems. The model consists of the vorticity balance of the depth-averaged flow and a hierarchy of equations for "vertical moments" of density and baroclinic velocity. The first vertical density moment is the (vertically integrated) potential energy, which is used to describe the predominant link between the barotropic and the baroclinic oceanic flow in the presence of sloping topography. Tendency equations for the vertical moments of density and baroclinic velocity and an appropriate truncation of the coupled hierarchy of moments are derived that, together with the barotropic vorticity balance, yield a closed set of equations describing the barotropic-baroclinic interaction (BARBI) model of the oceanic circulation. Idealized companion experiments with a numerical implementation of the BARBI model and a primitive equation model indicate that wave propagation properties and baroclinic adjustments are correctly represented in BARBI in midlatitudes as well as in equatorial latitudes. Furthermore, a set of experiments with a realistic application to the Atlantic/ Southern Ocean system reproduces important aspects that have been previously reported by studies of gyre circulations and circumpolar currents using full primitive equation models.
\end{abstract}

\section{Introduction}

It is a common approach to split the oceanic flow into a depth-independent part, called the barotropic flow, and the deviation, the baroclinic flow. This concept is considered to be useful under the premise that the barotropic flow is dominantly forced by the surface wind stress. However, the benefit of such a decomposition may depend on the "importance" (which might be large) and our "understanding" (which might not be as large) of the interaction between both components. In the vorticity balance for the large-scale, depth-averaged momentum the predominant coupling to the baroclinic field is given by torques generated by pressure gradients acting on an inclined bottom, sometimes called JEBAR (joint effect of baroclinicity and relief) torque (Sarkisyan and Ivanov 1971). JEBAR can be written as a torque involving gradients of the vertically integrated potential energy stored in the stratification and the topography

\footnotetext{
* Current affiliation: Institut für Meereskunde, Kiel, Germany.
}

Corresponding author address: Carsten Eden, Institut für Meereskunde, FB I, Theorie und Modellierung, Düsternbrooker Weg 20, 24105 Kiel, Germany.

E-mail: ceden@ifm.uni-kiel.de and can become one of the dominant terms in the barotropic vorticity balance (see, e.g., Holland 1973; Greatbatch et al. 1991; Böning et al. 1996).

It is the purpose of this study to present a new model concept, which we name BARBI (standing for barotropic-baroclinic interaction model). BARBI's major task is to implement the correct description of the interaction of barotropic flow and baroclinic flow in the presence of topography in a simplified ocean general circulation model. BARBI consists of and predicts vertical moments ${ }^{1}$ of density and velocity. The first density moment is identical to the vertically integrated potential energy, which we use (via the JEBAR torque) to couple the baroclinic to the barotropic flow. Together with certain closure assumptions, BARBI assembles therefore the essential dynamics of baroclinic circulation over topography and results in a simplified (with respect to the primitive equations) closed system describing the largescale wind- and buoyancy-driven oceanic circulation.

In its physical content, BARBI leans on the planetary geostrophic equations, although it does not suffer from

\footnotetext{
${ }^{1}$ We define a "vertical moment" of, say, $\phi$ as the vertical integral of $\phi$ multiplied with a power of depth, that is, $\int \phi z^{n} d z$, where $n$ is the order of the moment.
} 
a singularity at the equator. Its mathematical complexity is comparable with layered, reduced-gravity, or quasigeostrophic models since there are only a few two-dimensional equations in BARBI. The fundamental difference to existing models is the treatment of the vertical dependence of the baroclinic variables, namely, density and baroclinic velocity: the model equations are derived by vertical integration and projection of the primitive equations onto powers of depth $\left(z^{n}\right)$. In effect, the vertical coordinate is transformed by this procedure into the order of the vertical moments of the baroclinic variables. This treatment of the vertical dependence comes along as a natural consequence of the appearance of the first density moment (potential energy) in the JEBAR term of the barotropic vorticity equation. Furthermore, it naturally turns out that "baroclinic velocity moments" can be used to calculate the effects of advection by the baroclinic flow on the vertical density moments. As a consequence of the projection procedure, BARBI is given, in principle, by an infinite, coupled set of equations for vertical moments of increasing order. In practice, however, the coupled hierarchy can efficiently be truncated, as we shall show below, to the extent that only a couple of lower order moments are necessary to describe the essential dynamics of the baroclinic flow over topography.

Our new model concept competes, in a sense, with similar models with simplified physics. We want to stress, therefore, its major advantages:

- there is no restriction on topography, neither being confined to the lowest layer (as in layered, reducedgravity models ${ }^{2}$ ) nor being infinitesimal in terms of height or slope (as in quasigeostrophic models),

- there is no restriction to nonequatorial regions, as for quasigeostrophic or planetary geostrophic models, and

- the forcing of currents by surface buoyancy flux is explicitly included (in contrast to all other models with simplified physics mentioned above), though we do not make use of it in the current version of BARBI.

The most prominent feature of BARBI is that the fundamental role of the interaction between topography and baroclinicity in generating large-scale ocean transports is correctly represented in the model equations without any truncation. We think that, so far, the representation of topography has not attracted enough attention in models with simplified physics and that, therefore, BARBI may contribute to a better understanding of important ocean dynamics by means of simplified physics.

Of course, there are also disadvantages of BARBI to be mentioned. The effect of baroclinic advection of perturbation density on the vertical density moments is not as elegant and efficient to express in terms of vertical

\footnotetext{
${ }^{2}$ However, note that in some layered models-for example, recent versions of MICOM (Bleck et al. 1992), an "isopycnal" primitive equation model-layers of zero (or at least very small) thickness are defined to circumvent the artifacts of the "layered formulation."
}

moments of velocity and density as other effects. This effect is, however, small in the wave and wind-driven applications presented here (thermohaline-forced flow, in which this effect becomes important, will be considered in a companion paper in preparation). On the other hand, it is possible to derive approximate forms of the effect in an truncated BARBI model, utilizing an expansion of the vertical coordinate in the basis of modified Legendre polynomials, and the proposed truncation procedure. Using the same approach, it is in principle possible to relax almost all assumptions that we make in the present study, for example, the neglection of advection of momentum.

The paper is organized as follows. BARBI's hierarchy of equations is outlined in section 2 and summarized in section 3. For practical applications the (infinite) hierarchy must be truncated. We propose a truncation concept, based on wave solutions of the model, described in section 4. To test the new model concept, we apply a numerical implementation of BARBI to a number of circulation problems in section 5, namely propagation of disturbances in midlatitude and equatorial basins, the wind-driven circulation in a basin with topography, and some realistic wind-driven cases with an Atlantic model with attached Southern Ocean. Except for the last case, all BARBI experiments are compared with twin experiments with a primitive equation model (Pacanowski 1995; Redler et al. 1998). The last section is a concluding discussion of our findings. We have added three appendixes. In appendix A we outline a way to approximate the effect of the baroclinic advection of perturbation density in BARBI, in appendix B we cover simple parameterizations for dissipative and diabatic terms in BARBI, and in appendix $\mathrm{C}$ we discuss the general, flat-bottom, and topographic wave properties of the model.

\section{Derivation of BARBI}

We start with the Boussinesq form of the primitive equations. Vertical integration of the momentum balance yields the barotropic momentum balance: ${ }^{3}$

$$
\begin{aligned}
\frac{\partial \mathbf{U}}{\partial t}+f \mathbf{k} \times \mathbf{U}= & -\int_{-h}^{0} \nabla p d z+\boldsymbol{\tau}_{S}-\boldsymbol{\tau}(-h) \\
& +\int_{-h}^{0} \boldsymbol{\nabla} \cdot \mathbf{J}_{u} d z
\end{aligned}
$$

where $\mathbf{U}$ is the vertically integrated momentum, given by $\mathbf{U}=\int_{-h}^{0} \mathbf{u} d z$ (u is the total velocity), $h$ is ocean depth, and $p$ is the pressure (divided by a constant reference density). Stresses at the surface $\tau_{S}$ and the bottom $\boldsymbol{\tau}(-h)$ enter from the divergence of the vertical tur-

\footnotetext{
${ }^{3}$ All vectors are two dimensional horizontal, except for the following convention: $\mathbf{k}=(0,0,1)$ is a three-dimensional vertical unit vector, and $\mathbf{k} \times \mathbf{u}=\mathbf{k} \times(u, v, 0)=(-v, u, 0) \rightarrow(-v, u)$.
} 
bulent transports of momentum; $\mathbf{J}_{u}$ (a tensor) is the turbulent, lateral flux of momentum. Although advection of momentum can become an important baroclinic/barotropic coupling agent for mesoscale flows (and could be included in an approximative form), we neglect it here since we are mainly interested in large-scale flows.

The pressure gradient term in Eq. (1) can be decomposed as

$$
\begin{aligned}
\int_{-h}^{0} \boldsymbol{\nabla} p d z= & h \boldsymbol{\nabla}\left[p(z=0)+g \int_{-h}^{0} \rho d z\right] \\
& +\nabla\left(g \int_{-h}^{0} z \rho d z\right)
\end{aligned}
$$

using the hydrostatic relation $\partial p / \partial z=-g \rho$, where $\rho$ denotes in situ density (normalized by a constant reference density). Two types of pressure terms appear: the first term on the rhs is associated with the bottom pressure, while the second is the gradient of the vertically integrated potential energy. From Eq. (1) we may formulate the vorticity balance for the depth-averaged flow $\mathbf{U} / h$ :

$$
\begin{aligned}
\frac{\partial}{\partial t} \boldsymbol{\nabla} & \cdot \frac{1}{h} \boldsymbol{\nabla} \psi+\underline{\boldsymbol{\nabla}} \psi \cdot \boldsymbol{\nabla} \frac{f}{h} \\
= & -\frac{1}{h^{2}} \underline{\boldsymbol{\nabla}} E \cdot \underline{\boldsymbol{\nabla}} h \\
& +\underline{\boldsymbol{\nabla}} \cdot\left[\frac{1}{h} \boldsymbol{\tau}_{S}-\frac{1}{h} \boldsymbol{\tau}(-h)+\frac{1}{h} \int_{-h}^{0} \boldsymbol{\nabla} \cdot \mathbf{J}_{u} d z\right],
\end{aligned}
$$

where $\psi$ denotes the barotropic transport streamfunction defined by $\mathbf{U}=\boldsymbol{\nabla} \psi$ (the operator $\underline{\nabla}$ is shorthand for $\mathbf{k}$ $\times \nabla$; furthermore, $\boldsymbol{\nabla} A \cdot \nabla B$ is the Jacobian of $A$ and $B)$. Here we have made use of of the rigid-lid assumption and the volume conservation $\boldsymbol{\nabla} \cdot \mathbf{U}=0$. The bottom pressure term drops out and the JEBAR torque [first term on the rhs of Eq. (2)] shows up, involving the vertically integrated potential energy $E=g \int_{-h}^{0} z \rho d z$ as the remaining coupling to the baroclinic flow (note that the coupling due to the frictional terms, which are evaluated in appendix B, tend to be of minor importance and are consequently ignored here).

We proceed by formulating a tendency equation for the vertically integrated potential energy $E$ to close the balance in Eq. (2). It turns out that higher order vertical moments of density are needed as well. Thus we define

$$
E_{n}=g \int_{-h}^{0} z^{n} \rho d z, \quad n=0,1,2, \ldots
$$

Note that $E_{1}=E$. For simplification, the balances of heat and salt are combined into a thermohaline balance for density,

$$
\frac{\partial \rho}{\partial t}+\mathbf{u} \cdot \boldsymbol{\nabla} \rho+w\left(\frac{\partial \rho}{\partial z}-\frac{N^{2}}{g}\right)=-\frac{\partial B_{\rho}}{\partial z}-\nabla \cdot \mathbf{J}_{\rho} .
$$

Here, $\mathbf{u}$ and $w$ are the (total) horizontal and vertical velocities, $B_{\rho}$ and $\mathbf{J}_{\rho}$ vertical and horizontal density fluxes, respectively, which cover all diabatic process, for example, convection, mixing, and stirring by eddy activity and sources of heat and salt at the surface. Note that we have neglected effects of compressibility in Eq. (3), which puts the thermohaline balance in BARBI on a similar footing as usually implemented in simplified dynamics (e.g., quasigeostrophic or layered, reducedgravity models) where density is replaced by a potential (or "neutral") density. For the formulation of the diabatic terms in Eq. (3), it is necessary to assume furthermore a linear equation of state (which is, however, not necessary to derive the advective terms). The density $\rho$ is a deviation from a mean background profile of density $\bar{Q}(z)$, given by the Brunt-Väisälä frequency

$$
N^{2}(z)=-g \frac{d \bar{Q}(z)}{d z} .
$$

To distinguish between barotropic and baroclinic density advection, we split the total advective flow $\mathbf{u}$ in Eq. (3) into a barotropic part $\mathbf{U}$ and a baroclinic part $\mathbf{u}^{\prime}=\mathbf{u}$ - $\mathbf{U} / h$, with the corresponding barotropic $(W)$ and baroclinic $\left(w^{\prime}\right)$ vertical velocities, ${ }^{4}$

$$
\begin{aligned}
W & =\frac{z}{h^{2}} \mathbf{U} \cdot \boldsymbol{\nabla} h, \\
w^{\prime} & =-\boldsymbol{\nabla} \cdot \int_{-h}^{z} \mathbf{u}^{\prime} d z^{\prime} \quad \text { with } w=W+w^{\prime} .
\end{aligned}
$$

To obtain now a tendency equation for the vertical density moment $E_{n}$, we multiply Eq. (3) with $g$ and $z^{n}$ and integrate over depth, which yields

$$
\begin{aligned}
\frac{\partial E_{n}}{\partial t}+h^{n} \mathbf{U} \cdot \boldsymbol{\nabla} \frac{E_{n}}{h^{n+1}} & =\bar{S}_{n}+S_{n}^{\prime}+S_{n}^{\prime \prime}+Q_{n}+D_{n} \\
\bar{S}_{n} & =-h^{n} \mathbf{U} \cdot \boldsymbol{\nabla} \frac{g}{h^{n+1}} \int_{-h}^{0} z^{n} \bar{\varrho}(z) d z \\
S_{n}^{\prime} & =\int_{-h}^{0} z^{n} w^{\prime} N^{2}(z) d z \\
S_{n}^{\prime \prime} & =-g \int_{-h}^{0} z^{n}\left(\boldsymbol{\nabla} \cdot \mathbf{u}^{\prime} \rho+\frac{\partial w^{\prime} \rho}{\partial z}\right) d z .
\end{aligned}
$$

According to Eq. (4), changes in the vertical density moments $E_{n}$ are caused by advection of background density $\bar{\varrho}$ by the barotropic flow (given by $\bar{S}_{n}$ ), advection of $\bar{Q}$ by the baroclinic flow $\left(S_{n}^{\prime}\right)$ and advection of perturbation density $\rho$ by the baroclinic flow $\left(S_{n}^{\prime \prime}\right)$. The second term on the lhs in Eq. (4) results from horizontal

\footnotetext{
${ }^{4}$ They are derived from the total mass balance $\boldsymbol{\nabla} \cdot \mathbf{u}+\partial w / \partial z=0$ together with the kinematic boundary conditions (for a rigid lid) $w$ $=0$ at $z=0$ and $w+\mathbf{u} \cdot \boldsymbol{\nabla} h=0$ at $z=-h$.
} 
and vertical advection of $\rho$ by the barotropic flow. In addition to the advective sources, there are diabatic sources, decomposed into effects of vertical turbulent buoyancy flux $\left(Q_{n}\right)$ and lateral turbulent buoyancy fluxes $\left(D_{n}\right)$.

All source terms are evaluated and parameterized in the course of this work. We postpone the discussion of the diabatic sources $Q_{n}$ and $D_{n}$ and start with the advective terms. First, consider the effects of barotropic advection. Similar to the second term on the lhs in Eq. (4), it is evident that the effect of the lifting of the background mass field by the barotropic flow $\left(\bar{S}_{n}\right)$ can be expressed entirely in terms of $\mathbf{U}$ and the (given) background stratification $\bar{\rho}(z)$, in form of its corresponding vertical moments $\bar{E}_{n}=g \int_{-h}^{0} z^{n} \bar{Q}(z) d z$. Note that $\bar{E}_{n}$ depends only on $x$ and $y$ via the dependency on $h(x$, $y$ ). Therefore, $\bar{S}_{n} \neq 0$ only in regions with an inclined bottom. For, for example, a constant Brunt-Väisälä frequency $N=N_{0}$, the term takes the simple form

$$
\bar{S}_{n}=-(-1)^{n} \frac{N_{0}^{2} h^{n}}{n+2} \mathbf{U} \cdot \boldsymbol{\nabla} h .
$$

We want to point out, however, that the complete effect of vertical and horizontal barotropic advection of density on $E_{n}$ can be exactly derived for any choice of $\bar{Q}(z)$ or $N(z)$.

Next, we consider the source term $S_{n}^{\prime}$, representing the production of $E_{n}$ due to lifting of the background mass field by the baroclinic flow $\mathbf{u}^{\prime}$. For simplicity, we assume first, again, a constant Brunt-Väisälä frequency: $S_{n}^{\prime}$ is then given by

$$
S_{n}^{\prime}=\frac{N_{0}^{2}}{n+1} \nabla \cdot \int_{-h}^{0} z^{n+1} \mathbf{u}^{\prime} d z
$$

The form of $S_{n}^{\prime}$ suggests that the balance of baroclinic momentum $\mathbf{u}^{\prime}$ should be projected in the same way as Eq. (3) on $z^{n}$ to get prognostic equations for "baroclinic velocity moments." We follow this route and define the baroclinic velocity moments as

$$
\begin{aligned}
\mathbf{u}_{n+1}^{\prime}=\int_{-h}^{0} z^{n+1} \mathbf{u}^{\prime} d z \quad \text { for } n & =0,1,2, \ldots \text { and } \\
\mathbf{u}_{0}^{\prime} & =0
\end{aligned}
$$

and add them to our prognostic variables in BARBI. The baroclinic momentum balance is the difference between the complete momentum balance and the barotropic one, given by Eq. (1),

$$
\frac{\partial \mathbf{u}^{\prime}}{\partial t}+f \mathbf{k} \times \mathbf{u}^{\prime}=g \boldsymbol{\nabla} \int_{-h}^{z} \rho d z+\frac{1}{h} \boldsymbol{\nabla} E+\mathbf{F}^{\prime},
$$

with the baroclinic frictional force $\mathbf{F}^{\prime}$. The projection of the baroclinic momentum balance on $z^{n+1}$ yields

$$
\frac{\partial \mathbf{u}_{n+1}^{\prime}}{\partial t}+f \mathbf{k} \times \mathbf{u}_{n+1}^{\prime}
$$

$$
=-\frac{1}{n+2}\left[(-1)^{n} h^{n+1} \boldsymbol{\nabla} E_{1}+\boldsymbol{\nabla} E_{n+2}\right]+\int_{-h}^{0} z^{n+1} \mathbf{F}^{\prime} d z .
$$

A prognostic equation for the baroclinic velocity moments $\mathbf{u}_{n+1}^{\prime}$ is thus found, with a quite simple expression of the pressure gradient in terms of gradients of $E_{n}$. Note that no further assumptions have been made to derive Eq. (6) except that we have neglected advection of momentum as before. The divergence of $\mathbf{u}_{n+1}^{\prime}$ allows us in turn to calculate $S_{n}^{\prime}$ in the balance of $E_{n}$. However, at this point it turns out that, indeed, all (odd) vertical density moments are needed and not just $E_{1}$ since the interaction of potential energy $E_{1}$ with the baroclinic fields via $S_{1}^{\prime}$ (and thus $\mathbf{u}_{2}^{\prime}$ ) couples the third moment $E_{3}$ to the balance of $E_{1}$. For $E_{3}$ we need $S_{3}^{\prime}$, thus $\mathbf{u}_{4}^{\prime}$, therefore $E_{5}$, etc. Apparently, one needs a truncation of this coupled hierarchy at some order, which is discussed in section 4. Note also that starting with the balance of potential energy, only the odd moments $E_{n}(n=1,3$, $5, \ldots)$ are involved.

The source term $S_{n}^{\prime}$ can be evaluated for an arbitrary background density $\bar{\rho}(z)$, if this is expressed as a polynomial of depth; that is, $\bar{\rho}(z)=p_{1} z+p_{2} z^{2}+\cdots$. A truncated set of the expansion coefficients $p_{n}$ can be found for practical purposes by, for example, a simple fit to a profile of $\bar{\rho}(z)$, given at discrete depths. Using this expansion, we get for $S_{n}^{\prime}$, after some algebra which is suppressed here, the following expression:

$$
S_{n}^{\prime}=-g \sum_{j=1}^{\infty} \frac{j p_{j}}{j+n} \nabla \cdot \mathbf{u}_{n+j}^{\prime} .
$$

Hence, for a nonconstant $N(z)$, the term $S_{n}^{\prime}$ takes a similar form as for constant $N$. For the general case, however, depending on the form of $N(z)$ divergences of baroclinic velocity moments of higher order than $n+1$ (odd and even) might appear in $S_{n}^{\prime}$ (note that we get the constant- $N$-form of $S_{n}^{\prime}$ using $p_{1}=-N_{0}^{2} / g$ and $p_{n>1}=$ $0)$. In principle, the entire hierarchy beyond $n+1$ is involved in $S_{n}^{\prime}$ for an infinite expansion of $\bar{\rho}(z)$. A consequence for the model is that, in contrast to the case with constant $N$, odd and even $(>2)$ density moments are coupled through $S_{n}^{\prime}$.

Last, we have to evaluate the source term $S_{n}^{\prime \prime}$, stemming from advection (both vertical and horizontal) of perturbation density by the baroclinic flow. It is easy to show by scaling analysis that $S_{n}^{\prime \prime}$ is small compared to $S_{n}^{\prime}$ and $\bar{S}_{n}$, as long as the density perturbation $\rho$ is small compared to the background density $\bar{\rho}(z)$. In the wave and wind-driven experiments presented in section 5 , the $S_{n}^{\prime \prime}$ terms are indeed very small (they have been monitored in corresponding experiments with a primitive equation model). However, the term $S_{n}^{\prime \prime}$ might become large if the density perturbation becomes large, as, for example, in configurations with strong thermohaline forcing. We postpone the detailed treatment of the $S_{n}^{\prime \prime}$ to a companion paper in preparation but include a brief 
outline in appendix A where we show that, in effect, $S_{n}^{\prime \prime}$ can be expressed in terms of the density moments $E_{k}$ and baroclinic velocity moments $\mathbf{u}_{k}^{\prime}$.

\section{The BARBI model}

At this stage, BARBI is completed. No further variables will be added. However, some unspecified terms (of frictional and diabatic nature) have not yet been treated; they are discussed in appendix B and the results are used here. To summarize, BARBI consists of the following variables and corresponding tendency equations:

- the barotropic transport $\mathbf{U}=\int_{-h}^{0} \mathbf{u} d z=\underline{\nabla} \psi$ given by the barotropic vorticity balance

$$
\begin{aligned}
\frac{\partial}{\partial t} \boldsymbol{\nabla} & \cdot \frac{1}{h} \boldsymbol{\nabla} \psi+\underline{\nabla} \psi \cdot \nabla \frac{f}{h} \\
= & -\frac{1}{h^{2}} \underline{\nabla} E_{1} \cdot \boldsymbol{\nabla} h+\underline{\boldsymbol{\nabla}} \cdot \frac{\tau_{S}}{h} \\
& +A_{h} \boldsymbol{\nabla} \cdot\left(\frac{1}{h} \boldsymbol{\nabla} \cdot h \boldsymbol{\nabla}\right) \frac{1}{h} \boldsymbol{\nabla} \psi,
\end{aligned}
$$

- the vertical density moments $E_{n}=g \int_{-h}^{0} z^{n} \rho d z$ given by

$$
\begin{gathered}
\frac{\partial E_{n}}{\partial t}+h^{n} \mathbf{U} \cdot \boldsymbol{\nabla} \frac{E_{n}}{h^{n+1}}+(-1)^{n} \frac{N_{0}^{2} h^{n}}{n+2} \mathbf{U} \cdot \boldsymbol{\nabla} h \\
=\frac{N_{0}^{2}}{n+1} \boldsymbol{\nabla} \cdot \mathbf{u}_{n+1}^{\prime}+K_{h} \nabla^{2} E_{n}+Q_{n}
\end{gathered}
$$

for $n=0,1,2, \ldots$ (here written for the case of $N^{2}$ $=N_{0}^{2}=$ const and $S_{n}^{\prime \prime} \equiv 0$ ), and

- the baroclinic velocity moments $\mathbf{u}_{n+1}^{\prime}=\int_{-h}^{0} z^{n+1} \mathbf{u}^{\prime} d z$ given by

$$
\begin{aligned}
\frac{\partial \mathbf{u}_{n+1}^{\prime}}{\partial t} & +f \mathbf{k} \times \mathbf{u}_{n+1}^{\prime} \\
= & -\frac{1}{n+2}\left[(-1)^{n} h^{n+1} \nabla E_{1}+\nabla E_{n+2}\right] \\
& +A_{v} n(n+1) \mathbf{u}_{n-1}^{\prime}+\frac{(-1)^{n} h^{n+1}}{n+2} \boldsymbol{\tau}_{S} \\
& +A_{h} \nabla^{2} \mathbf{u}_{n+1}^{\prime}
\end{aligned}
$$

for $n=0,1,2, \cdots$.

Horizontal and vertical viscosity $A_{h}$ and $A_{v}$ are used as well as a lateral diffusivity $K_{h}$. We are using no slip as the lateral boundary condition for momentum translating to similar conditions for $\mathbf{U}$ and $\mathbf{u}_{n+1}^{\prime}$, free slip at the bottom as worked out in appendix B, and von Neumann condition for $E_{n}$ (no buoyancy flux through lateral boundaries and the bottom). Surface boundary forcing enters through the wind stress $\tau_{S}$ and the surface density flux $Q_{n}$.
All variables in BARBI are two-dimensional. The vertical coordinate is transformed into the order of the vertical moments of the baroclinic variables, namely density and baroclinic velocity. This approach is a natural consequence of the occurrence of $E_{1}$ (potential energy) in the barotropic vorticity equation (JEBAR term) and $\mathbf{u}_{n+1}^{\prime}$ in the tendency equation for $E_{n}$. At the present stage, BARBI is neglecting advection of momentum and effects of compressibility or the nonlinearity of the equation of state and assumes that $\rho$ remains small when compared with $\bar{\varrho}$ (for $S_{n}^{\prime \prime} \ll S_{n}^{\prime}, \bar{S}_{n}$ ).

\section{Closure of the hierarchy of moments}

For practical purposes, we need a truncation of the coupled hierarchy of the vertical moments. We begin with the case of a constant $N$, a straightforward extension of the closure for nonconstant $N(z)$ is demonstrated afterward. A parameterization of $E_{L+2}$ in terms of $E_{n \leq L}$ is presented since, for constant $N$, we need $E_{L+2}$ in the pressure gradient forcing of $\mathbf{u}_{L+1}^{\prime}$, showing up in turn in the equation for $E_{L}$. Consideration of wave properties in BARBI is our guide for the closure. Therefore, we aim to construct the parameterization such that a truncated model has the correct gravity and geostrophic wave speeds.

It seems obvious that BARBI has barotropic and baroclinic geostrophic waves (associated with the planetary $\beta$ and topographic slopes) and baroclinic gravity waves. We want to note, here in passing, that it is possible to gain the correct dispersion relations for all baroclinic (flat bottom and topographic) waves (and baroclinic modes) in the limit of infinite number of vertical modes in BARBI. This is demonstrated in appendix C.

However, we start with a one-mode BARBI model for which the baroclinic equations (excluding effects of friction, horizontal advection, and diabatic sources) are given by

$$
\begin{aligned}
& \frac{\partial \mathbf{u}_{2}^{\prime}}{\partial t}+f \mathbf{k} \times \mathbf{u}_{2}^{\prime}=-\frac{1}{3} \nabla E_{1} h^{2}(\gamma-1), \\
& \frac{\partial E_{1}}{\partial t}-\frac{N_{0}^{2}}{2} \nabla \cdot \mathbf{u}_{2}^{\prime}=0 .
\end{aligned}
$$

Here, the parameterization $E_{3}=\gamma h^{2} E_{1}$ is used, and we have to find now a proper value for the closure parameter $\gamma$, which for simplicity is taken as a constant.

If we restrict Eq. (9) to an $f$ plane, the dispersion relation of inertio-gravity waves [proportional to expi( $\boldsymbol{\kappa}$ $\cdot \mathbf{x}-\omega t)]$ is obtained,

$$
\omega^{2}=f_{0}^{2}+c^{2} \kappa^{2} \quad \text { with } c^{2}=\frac{1-\gamma}{6}\left(N_{0} h\right)^{2},
$$

where $\boldsymbol{\kappa}=\left(\kappa_{1}, \kappa_{2}\right)$ denotes the wave vector and $c$ the gravity wave speed. We can choose $\gamma$ to gain the correct phase speed $c=N_{0} h / \pi$ of the first-mode gravity wave 
speed. ${ }^{5}$ This is achieved with $(1-\gamma) / 6=1 / \pi^{2}$ or $\gamma$ $\approx 0.3921$.

For the following reason, we suppress here any discussion of other wave branches. Equation (9) has indeed the form of the (reduced gravity) shallow water equations, since with the pressure variable $P=(\gamma-1) h^{2} E_{1} /$ 3 , we get

$$
\begin{gathered}
\frac{\partial \mathbf{u}_{2}^{\prime}}{\partial t}+f \mathbf{k} \times \mathbf{u}_{2}^{\prime}=-\nabla P, \\
\frac{\partial P}{\partial t}+c^{2} \boldsymbol{\nabla} \cdot \mathbf{u}_{2}^{\prime}=0 .
\end{gathered}
$$

Therefore, it is evident that our setting for $\gamma$ also yields the correct dispersion relations for the entire baroclinic wave family (as, e.g., short and long Rossby waves) in this one-mode BARBI model, including the spectra of equatorial waves. Boundary waves such as Kelvin and pseudo-Kelvin waves are implemented as well [the latter appear in numerical models, which do not resolve the first internal Rossby radius of deformation (see Davey et al. 1983; Hsieh et al. 1983; Killworth 1985)].

It is straightforward to extend the truncation procedure for BARBI models with more than one baroclinic mode. We propose the following closure for $E_{L+2}$ (in a model with $E_{1}, E_{3}, \ldots E_{L}$ )

$$
E_{L+2}=\sum_{j=1}^{L} \gamma_{j} E_{j} h^{L-j+2} .
$$

A wave solution $E_{n}=e_{n} h^{n-1} \operatorname{expi}(\boldsymbol{\kappa} \cdot \mathbf{x}-\omega t)$ yields for the (truncated) hierarchy of moments in this ( $L$ mode) BARBI model for the gravity wave branch $(f$ plane)

$$
\begin{aligned}
\frac{1}{6}\left(e_{3}-e_{1}\right) & =-\frac{\omega^{2}-f_{0}^{2}}{N_{0}^{2} h^{2} \kappa^{2}} e_{1} \\
\frac{1}{20}\left(e_{5}-e_{1}\right) & =-\frac{\omega^{2}-f_{0}^{2}}{N_{0}^{2} h^{2} \kappa^{2}} e_{3} \\
& \vdots \\
\frac{1}{(L+1)(L+2)}\left(e_{L+2}-e_{1}\right) & =-\frac{\omega^{2}-f_{0}^{2}}{N_{0}^{2} h^{2} \kappa^{2}} e_{L},
\end{aligned}
$$

which can be formulated in matrix form as

$$
\mathbf{M}\left(\gamma_{j}\right) \mathbf{e}=\frac{\omega^{2}-f_{0}^{2}}{N_{0}^{2} h^{2} \kappa^{2}} \mathbf{e}, \quad \mathbf{e}=\left(e_{1}, e_{3}, \ldots, e_{L}\right)^{\mathrm{T}}
$$

since

$$
e_{L+2}=\sum_{j} \gamma_{j} e_{j},
$$

where $\mathbf{M}$ is an $L \times L$ matrix and $\mathbf{e}$ is an $L \times 1$ vector.

\footnotetext{
${ }^{5}$ For constant $N=N_{0}$, constant $h$, and a rigid lid the baroclinic eigenmodes are $\phi^{\nu}(z)=\cos \nu \pi z / h$ with eigenvalues $(\nu \pi)^{2}$, Rossby radii $N_{0} h /(|f| \nu \pi)$, and gravity wave speeds $N_{0} h /(\nu \pi)$, with $\nu=1$, $2, \ldots$.
}

For example, for long Rossby waves ( $\beta$ plane, $\partial \mathbf{u}_{n+1}^{\prime} / \partial t$ $\equiv 0$ ) we get a similar result with the same matrix $\mathbf{M}$ as before:

$$
\mathbf{M}\left(\gamma_{j}\right) \mathbf{e}=\frac{\omega f^{2}}{\beta N_{0}^{2} h^{2} \kappa_{1}} \mathbf{e} .
$$

Therefore, the dispersion relations $\omega=\omega(\boldsymbol{\kappa})$ for the different wave branches are determined by the eigenvalues $\zeta_{\nu}$ of $\mathbf{M}\left(\gamma_{j}\right)$. Without giving further details, we just note here that it is possible to adjust the eigenvalues by tuning the values of the $\gamma_{j}$, such that they become $\zeta_{\nu}=-1 /(\nu \pi)^{2}$ and therefore fit the (known) gravity wave and Rossby wave speeds (the problem is linear in $\gamma_{j}$ and can readily be solved). In consequence, the BARBI model will have as many baroclinic modes as vertical moments. Furthermore, the density moments resemble amplitudes of baroclinic modes in the basis of the (left) eigenvectors $\mathbf{e}_{v}($ a $1 \times L$ vector $)$ of $\mathbf{M}$. In other words, the components of $\mathbf{e}_{\nu}$ give coefficients for a linear combination of all $E_{n} / h^{n-1}$, to filter out the $\nu$ th baroclinic mode (where $\boldsymbol{\nabla} h \equiv 0$ ). We use the eigenvectors $\mathbf{e}_{\nu}$ in section 5 for the analysis of results of numerical models (both BARBI and a primitive equation model).

Next, we briefly outline the consequences of a nonconstant $N$ to the truncation procedure at order $L$. In contrast to the case with constant $N$, the term $S_{n}^{\prime}$ involves now higher order $(>L+1)$ baroclinic velocity moments, which, in addition to $E_{L+2}$, have to be parameterized.

Consider again a one-mode BARBI model, where we now parameterize all higher order $(n>1)$ density moments:

$$
E_{n}=\gamma h^{n-1}(-1)^{n-1} E_{1} \quad \text { for } n=2,3,4, \ldots
$$

Using this parameterization in the equations for the truncated $(n>2)$ baroclinic velocity moments, assuming a flat bottom and neglecting the $A_{v}$ term, we see that $\mathbf{u}_{j>2}^{\prime}$ can be expressed in terms of $\mathbf{u}_{2}^{\prime}$ :

$$
\mathbf{u}_{j}^{\prime}=\mathbf{u}_{2}^{\prime} \frac{3 h^{j-2}}{(j+1)}(-1)^{j} \quad \text { for } j=3,4,5, \ldots .
$$

Therefore, we get for the general case of a nonconstant $N$ in a one-mode model:

$$
S_{1}^{\prime}=-g \boldsymbol{\nabla} \cdot \mathbf{u}_{2}^{\prime}\left[\frac{p_{1}}{2}-3 \sum_{j=2}^{\infty} \frac{j p_{j} h^{j-1}(-1)^{j}}{(j+1)(j+2)}\right] .
$$

The parameter $\gamma$ is determined as before by solving the baroclinic wave equation, which now yields

$$
c^{2}=g h^{2}(\gamma-1)\left[\frac{p_{1}}{6}-\sum_{j=2}^{\infty} \frac{j p_{j} h^{j-1}(-1)^{j}}{(j+1)(j+2)}\right] .
$$

Given a profile for $\bar{Q}$, we can calculate the $p_{n}$ and get an "effective" constant $N_{0}^{2}$, which is given by the sum in square brackets involving the $p_{n}$. The correct gravity 


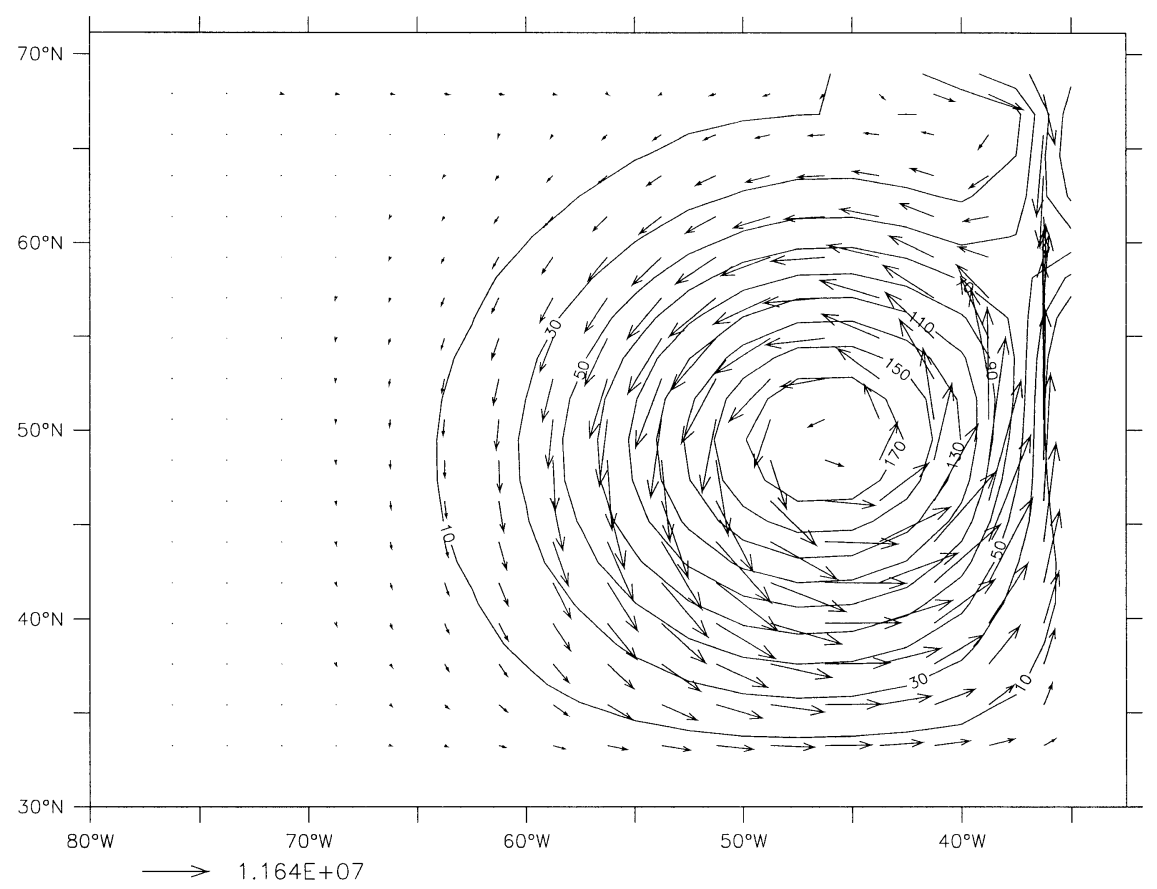

FIG. 1. The first density moment and first baroclinic velocity moments (arrows) in BARBI after 30 days. Contour interval is $20 \mathrm{~m}^{2} \mathrm{~s}^{-2}$.

wave speed ${ }^{6} c$, determined by the actual profile of $N(z)$, can be tuned with the choice of $\gamma$.

The truncation approach is readily extended to more than one mode in a model with nonconstant $N$. For an $L$-mode BARBI model, we are using the parameterization

$$
E_{n}=(-1)^{n-1} \sum_{j=1}^{L} \gamma_{j} E_{j} h^{n-j} \text { for } n>L .
$$

Then, we can express the truncated, higher-order velocity moments as

$$
\mathbf{u}_{L+1+j}^{\prime}=\mathbf{u}_{L+1}^{\prime} \frac{L+2}{L+2+j} h^{j}(-1)^{j} .
$$

We can use this expression for the evaluation of $S_{n}^{\prime}$ for the case of a nonconstant $N$ in an $L$-mode model.

\section{Numerical experiments}

We have assessed the performance of BARBI by comparison of integrations of a numerical implementation of BARBI with integrations of a primitive equation model (called MOM hereinafter; Pacanowski 1995; Redler et al. 1998) in various model setups. The numerical BARBI model is very similar to MOM (most code fragments have actually been taken from MOM); for example, finite differences in space and time are

\footnotetext{
${ }^{6}$ To our surprise, it came out that the optimal parameter $\gamma$ is approximately given by $\gamma=1-6 / \pi^{2}$ (the same optimal $\gamma$ as for a constant $N^{2}$ ), for any background density profile that we have tested.
}

second order and an Arakawa B grid is used. The approximations currently used in BARBI have been tested by evaluation of the appropriate terms in the numerical experiments. In particular, we have verified that the linearization of the momentum balances and the neglection of $S_{n}^{\prime \prime}$ in the density moment balances is appropriate since these effects are present in MOM. It turns out that both assumptions are valid.

In order to present the results of the integrations, we choose and discuss here results from experiments in four different configurations:

- wave propagation in a flat bottom, midlatitude basin on a $\beta$ plane, initialized with a Gaussian disturbance of the first moments near the eastern boundary of the basin (expt DISTURB);

- spinup of an equatorial $\beta$ plane circulation, forced with wind stress in the western part of the basin (expt EQUAT);

- a midlatitude basin on a $\beta$ plane with a meridional ridge, forced with wind stress (expt RIDGE); and

- a realistic model of the Atlantic and the Antarctic Circumpolar Current (expt ATLACC).

\section{a. Experiment DISTURB}

The initial condition for experiment DISTURB is a Gaussian perturbation in the first density moments near the eastern boundary, as shown in Fig. 1. BARBI uses truncation at order 5, with $E_{5}=0.66 h^{2} E_{3}$ such that $E_{1}$ and $E_{3}$ are predicted and the lowest two baroclinic 
modes are present. In addition, we discuss as well a one-mode BARBI model, using $E_{3}=0.3921 h^{2} E_{1}$. The vertical resolution of the primitive equation model is 10 $\mathrm{m}$ thick at the top increasing to $250 \mathrm{~m}$ at the bottom (at $5500 \mathrm{~m}$ ), altogether 45 vertical levels. Both models use a $\beta$ plane of $40^{\circ}$ in latitudinal range referenced to $30^{\circ} \mathrm{N}$, which is also the latitude of the southern boundary of the domain. The zonal resolution is $2.5^{\circ} \cos \phi$ and the meridional resolution is $2.5^{\circ} \cos 30^{\circ}$. The horizontal viscosity is $2 \times 10^{5} \cos \phi \mathrm{m}^{2} \mathrm{~s}^{-1}$, the vertical viscosity is $10^{-3} \mathrm{~m}^{2} \mathrm{~s}^{-1}$, the horizontal diffusivity is $2000 \cos \phi \mathrm{m}^{2}$ $\mathrm{s}^{-1}$, and the vertical diffusivity is $10^{-4} \mathrm{~m}^{2} \mathrm{~s}^{-1}$. The bottom is flat, and bottom friction is set to zero. The background stratification is the same in both models, given by a constant stability frequency $N_{0}=2.6 \times 10^{-3}$ $\mathrm{s}^{-1}$. The time step is $1 \mathrm{~h}$ in both models. We are using a linear equation of state in MOM with $\partial \rho / \partial T=-0.2$ $\mathrm{kg} \mathrm{m}^{-3} \mathrm{~K}^{-1}$; salinity is kept constant. The perturbation in $E_{1}$ is incorporated into MOM with a Gaussian perturbation in temperature, decaying from about $0.5 \mathrm{~K}$ at the surface to the abyssal ocean with a vertical $e$-folding scale of $500 \mathrm{~m}$.

Both models show a westward propagation of the perturbation in the baroclinic structure. Figure 2 displays this behavior along a latitude centered in the middle of the basin. The initially positive $E_{1}$ anomaly splits up into two signals with opposite sign. The positive anomaly moves westward with the speed of the first baroclinic (long) Rossby wave in the model setup, while the negative anomaly propagates with the phase speed of the second baroclinic mode (the mode speeds are indicated by the dashed lines in Fig. 2). A very similar signal shows up in $E_{3}$ in both MOM and the two-mode BARBI (not shown). Figure 2c shows the result of the experiment with the one-mode BARBI model. The first mode Rossby wave speed is very similar to MOM and the two-mode model, but in the one-mode BARBI model the negative anomaly in $E_{1}$ with propagation speed of the second baroclinic Rossby wave is absent.

In addition to the Rossby waves, MOM and BARBI exhibit boundary wavelike structures (pseudo-Kelvin wave: see Davey et al. 1983; Hsieh et al. 1983; Killworth 1985). They show up first at the eastern boundary, where the easternmost part of the initial Gaussian anomaly is detached from the interior anomaly and begins to propagate northward. An early stage (after 30 days of the integration) of this propagation can be seen in Fig. 1 . The speed of the propagation of the viscous waves, very similar in both MOM and BARBI, is in between the (slow) first baroclinic Rossby wave speed and the (fast) first-mode baroclinic Kelvin wave speed. Figure 3 shows these boundaries waves traveling along the northern boundary in the first year of the experiment. Again a separation of the signal into faster traveling positive amplitudes (first mode) and slower negative amplitudes (second mode) can be seen.

In summary, DISTURB shows that a low-order (one or two baroclinic modes) BARBI model is able to sim-

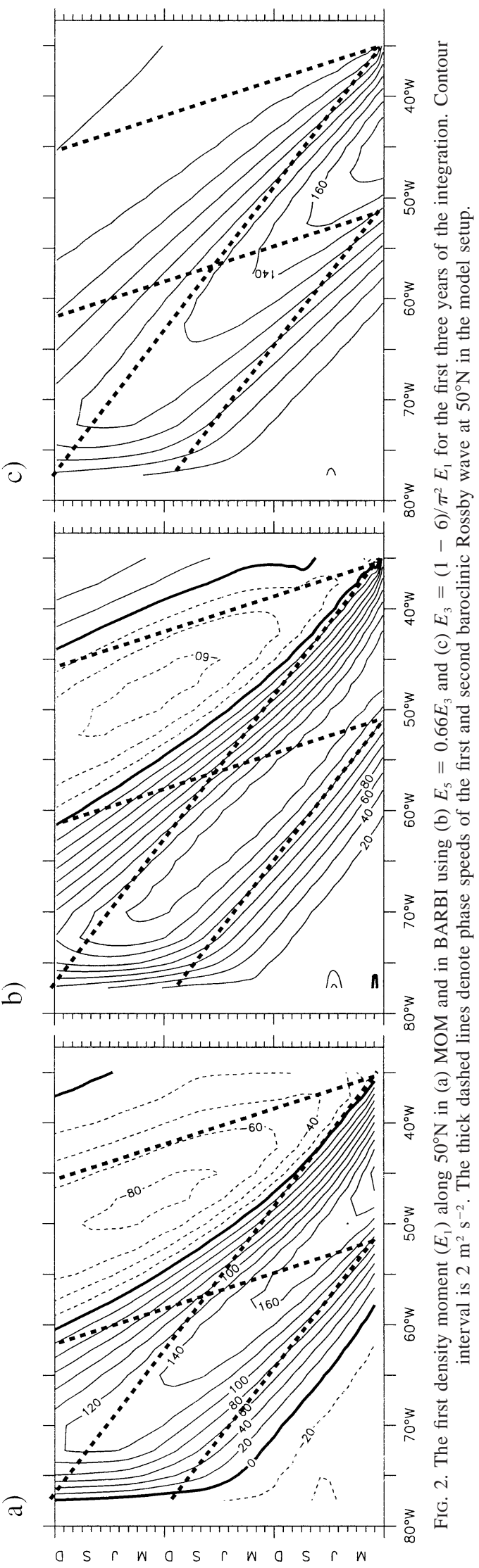



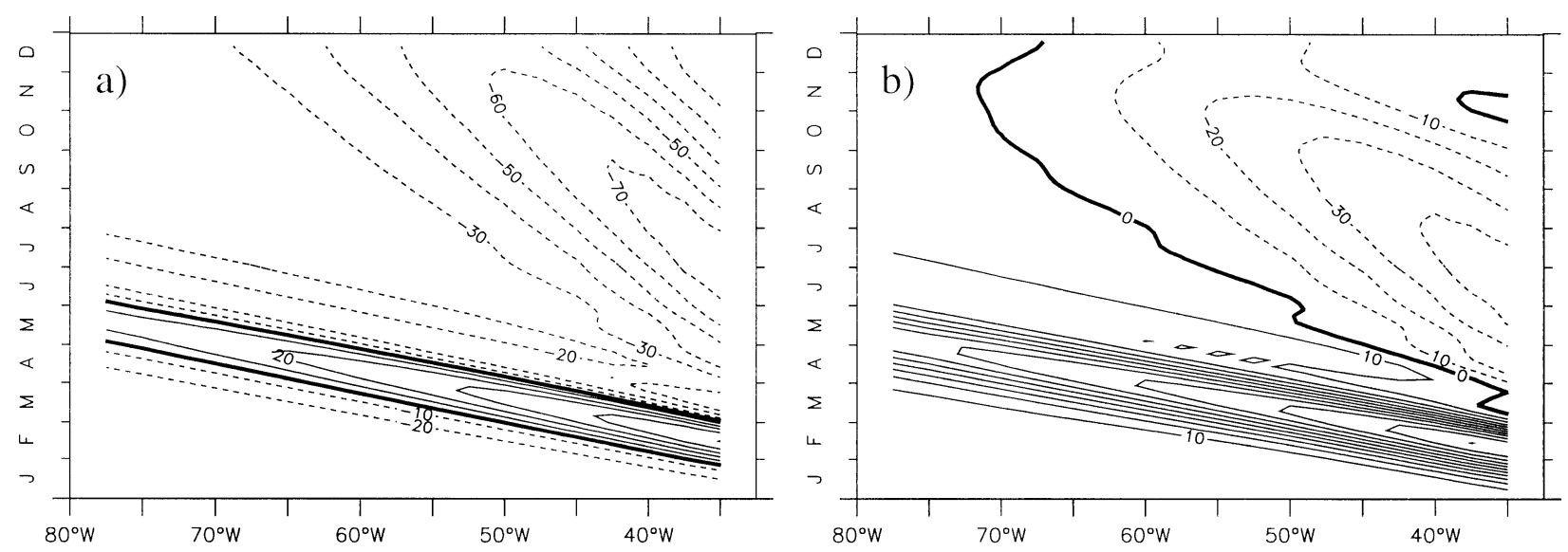

FIG. 3. The first density moment $\left(E_{1}\right)$ along the northern boundary (a) in MOM and (b) in BARBI. Contour interval is $20 \mathrm{~m}^{2} \mathrm{~s}^{-2}$.

ulate the important aspects of (geostrophic) wave propagation in a flat-bottom ocean, which are present in the much more complicated primitive equation model, confirming the aim of our truncation procedure.

\section{b. Experiment EQUAT}

Experiment EQUAT treats the spinup of the baroclinic circulation in an equatorial basin, and mimics the oceanic dynamics in the onset of an ENSO event in the tropical Pacific. The parameters for both models are the same as in DISTURB, except that we choose here $N=$ $1.5 \times 10^{-3} \mathrm{~s}^{-1}$ and a horizontal resolution of $2^{\circ}$. The basin extent is $100^{\circ}$ in longitudinal direction from $14^{\circ} \mathrm{S}$ to $14^{\circ} \mathrm{N}$ on an equatorial $\beta$ plane. In addition, we apply open boundary conditions (Stevens 1990) for the baroclinic and barotropic modes in MOM and BARBI at the northern and southern boundaries to allow (Kelvin) waves to propagate out of the domain (otherwise they would travel rapidly along the boundaries and affect the equatorial region again). Starting from a state of rest, the models are forced with a westerly wind stress to the west of $60^{\circ} \mathrm{W}$ with an amplitude of $0.06 \mathrm{~N} \mathrm{~m}^{-2}$. East of $60^{\circ} \mathrm{W}$ the wind stress is zero.

For BARBI we choose a truncation at order 7 with the closure $E_{7} / h^{6}=0.026174 E_{1}-0.379621 E_{3} / h^{2}+$ $1.207826 E_{5} / h^{4}$, such that three baroclinic modes are present. We show the results in the basis of the eigenvectors $\mathbf{e}_{v}$ of $\mathbf{M}\left(\gamma_{j}\right)$, as described in section 4 . Note that the scalar product $\mathbf{e}_{v} \cdot\left(E_{1}, E_{3} / h^{2}, E_{5} / h^{4}\right)$ is equivalent to the amplitude of the $\nu$ th baroclinic mode, whereas $E_{n}$ contains a mixture of amplitudes of all modes (as in the above example, Figs. 2 and 3). Figure 4 shows the baroclinic-mode amplitudes for both models along the equator during the first few months of the experiments. There is clearly an eastward-propagating equatorial Kelvin wave present in all modes and both models. While the first baroclinic Kelvin wave travels in about 1.5 months across the basin, the second-mode wave needs about 3.5 and the third about 6 months. The am- plitude of the first baroclinic mode is the largest, while the amplitude of the third mode is the smallest (note the different contour intervals in the figure). Wave speeds and amplitudes are almost identical in both models.

As already indicated in Fig. 4, the equatorial Kelvin waves are reflected at the eastern boundary as equatorial Rossby waves, propagating westward again and Kelvin waves, propagating north- and southward. As a consequence of our choice of $N$, the largest amplitudes of the Rossby wave show up symmetrically at about $8^{\circ}-$ $10^{\circ}$ north and south of the equator. Again wave speeds and amplitudes agree well between BARBI and MOM. According to Fig. 4, the first (second, third) mode Rossby wave reaches again the forcing region after about 6 months (1 year, 7.5 years) after eastern reflection. In summary, EQUAT confirms that with respect to important aspects of wave propagation, BARBI and the truncation procedure performs equally well in equatorial regions as in midlatitudes.

\section{c. Experiment RIDGE}

RIDGE is the same model configuration as DISTURB, with the difference that both models are now forced with wind stress and topography is present in form of a ridge. A cosine-shaped, zonal wind stress (with maximal amplitude of $0.6 \times 10^{-4} \mathrm{~m}^{2} \mathrm{~s}^{-2}$ ) yields the classical double-gyre solution in the flat bottom case (not shown) with a western boundary transport of about $14 \mathrm{~Sv}\left(\mathrm{~Sv} \equiv 10^{6} \mathrm{~m}^{3} \mathrm{~s}^{-1}\right)$. The topography, shown in Fig. 5, is a shallow north-south ridge in the middle of the basin.

Figures $6 \mathrm{a}$ and $6 \mathrm{~b}$ show the initial response of the barotropic streamfunction $\psi$ to the forcing in experiment RIDGE for MOM and BARBI. Obviously, a signature of the topography is present at the early stages. Since the bottom (JEBAR) torques are initially very small (not shown), the topographic Sverdrup solution is established (see, e.g., Schulman and Niiler 1970): any flow across 
Equatorial Kelvin waves
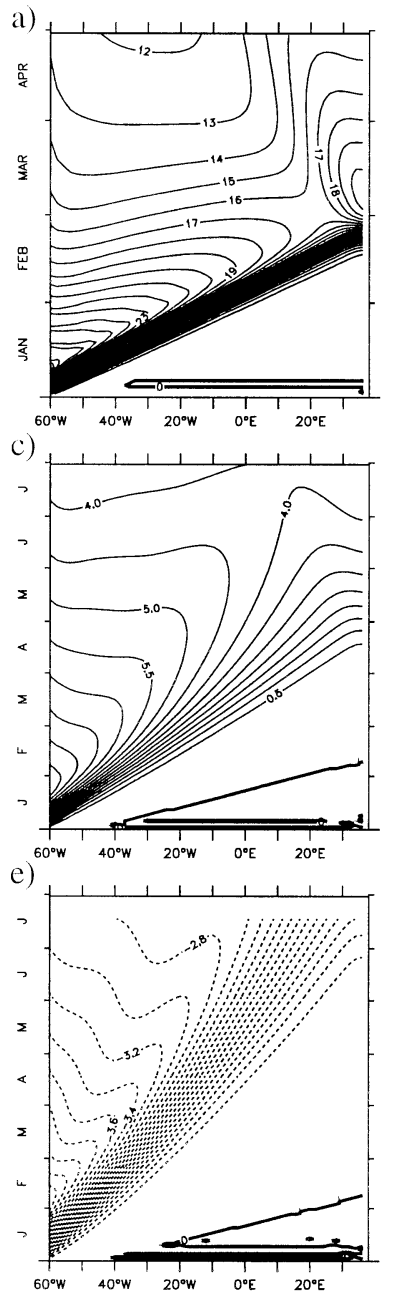

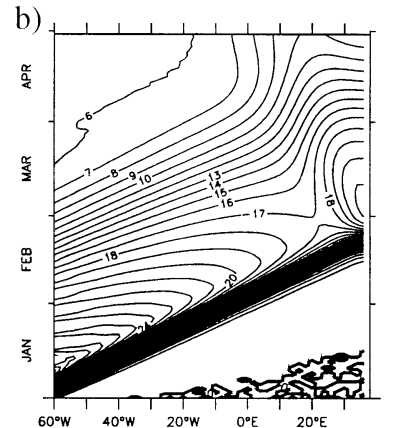

d)

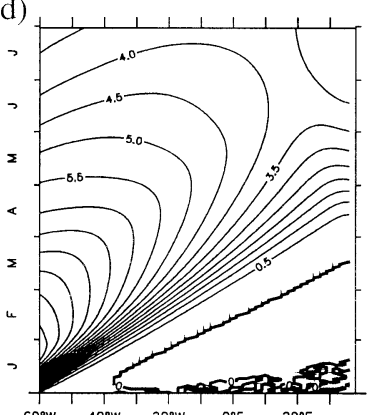

f)

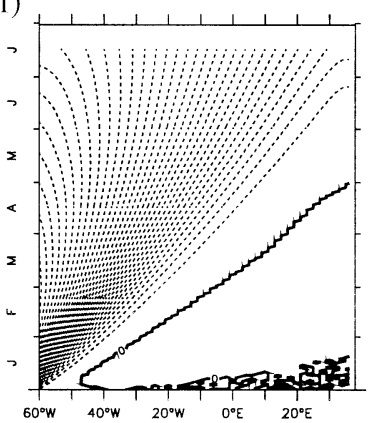

Equatorial Rossby waves

a)

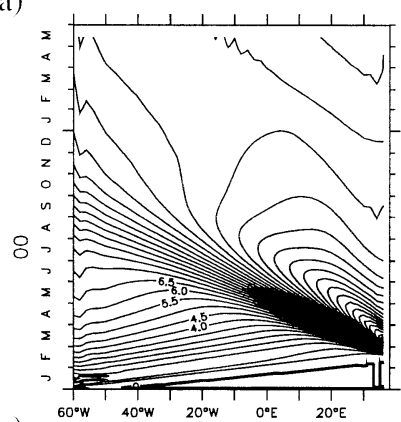

c)

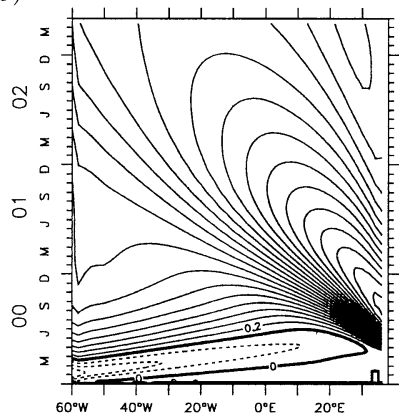

e)

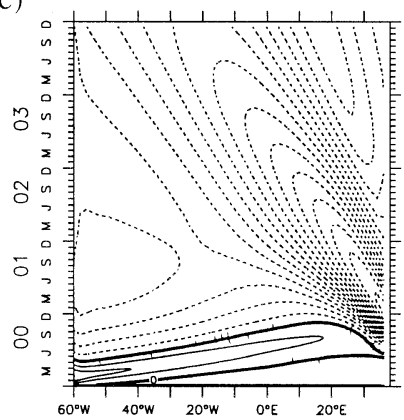

b)

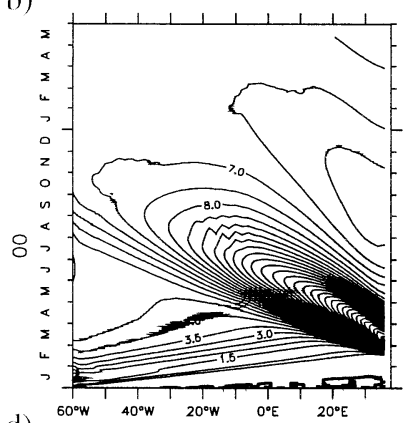

d)

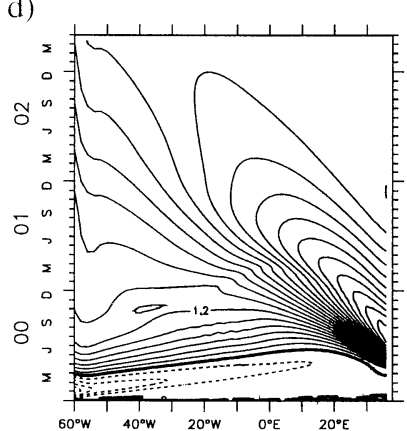

f)

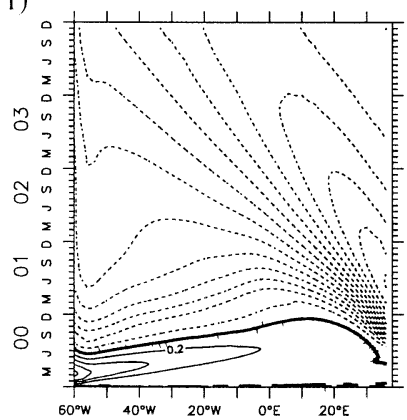

FIG. 4. (left) Equatorial Kelvin waves in (a), (c), (e) MOM and (b), (d), (f) BARBI. Shown is a section at the equator (excluding the region in the western part of the basin in which the wind stress forcing is applied) of the (a), (b) first, (c), (d) second, and (e), (f) third baroclinic modes for the first few months of the experiments. (right) Equatorial Rossby waves in (a), (c), (e) MOM and (b), (d), (f) BARBI. Shown is a section at $8^{\circ} \mathrm{N}$ with the same splitting. Contour interval is $1 \mathrm{~m}^{2} \mathrm{~s}^{-2}$ for the first mode, $0.5 \mathrm{~m}^{2} \mathrm{~s}^{-2}$ for the second mode, and 0.2 $\mathrm{m}^{2} \mathrm{~s}^{-2}$ for the third mode.

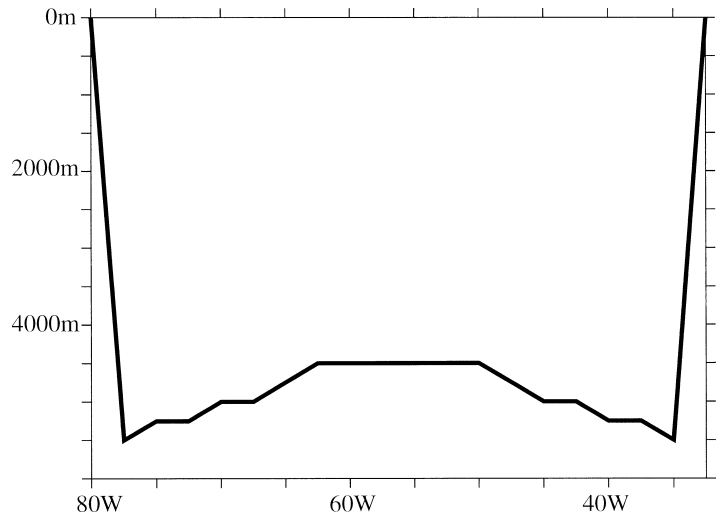

FIG. 5. The topography used for BARBI and the primitive equation model in experiment RIDGE. It is constant in the latitudinal direction. the geostrophic contours $f / h$ must be forced by the depth-modified wind stress curl $\boldsymbol{\nabla} \cdot \boldsymbol{\tau}_{S} / h$ or by friction. Note that without coupling of the barotropic and the baroclinic modes [i.e., by neglecting $\nabla E_{1} \cdot \nabla h$ in the vorticity balance Eq. (2)], there would be no further change in the barotropic mode in both models after about 1 week (approximately the passage time of the barotropic Rossby waves).

However, as a consequence of the increasing importance of the bottom torques in the vorticity balance, the streamfunction approaches the flat-bottom Sverdrup solution in the subsequent integration of both MOM and BARBI. Figures $6 \mathrm{c}$ and $6 \mathrm{~d}$ show the streamfunction after 12 months, when this process is nearly completed in both models. Both solutions are similar to each other and almost the same as the flat-bottom solution (not 
a)

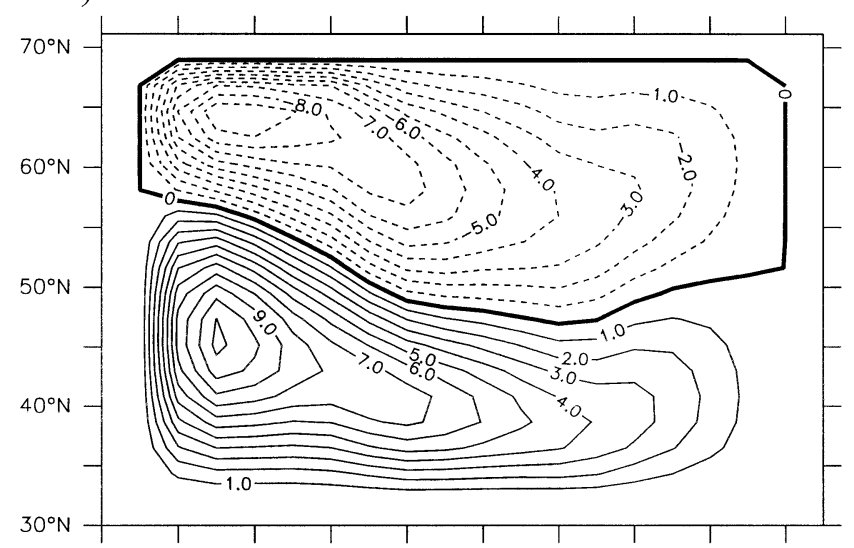

c)

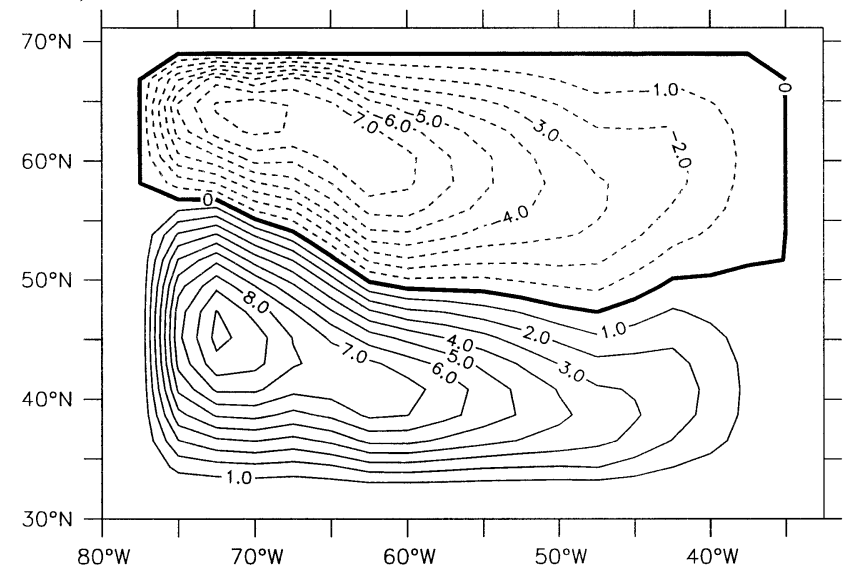

b)

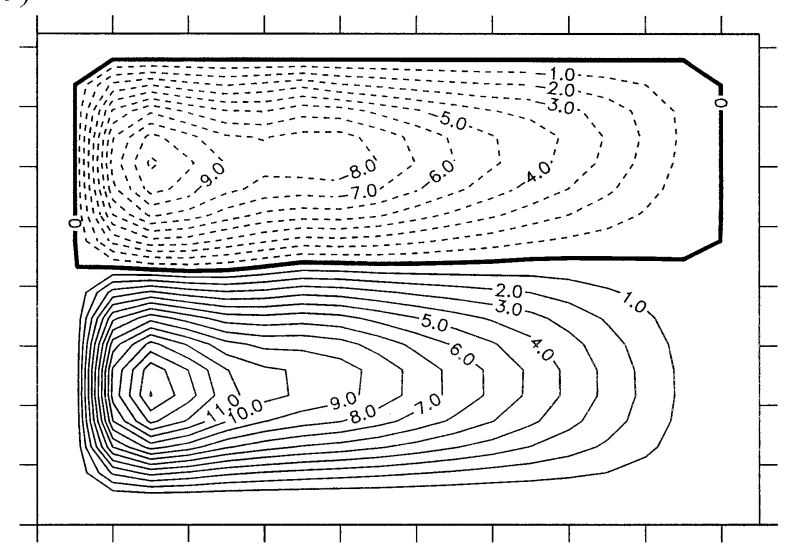

d)

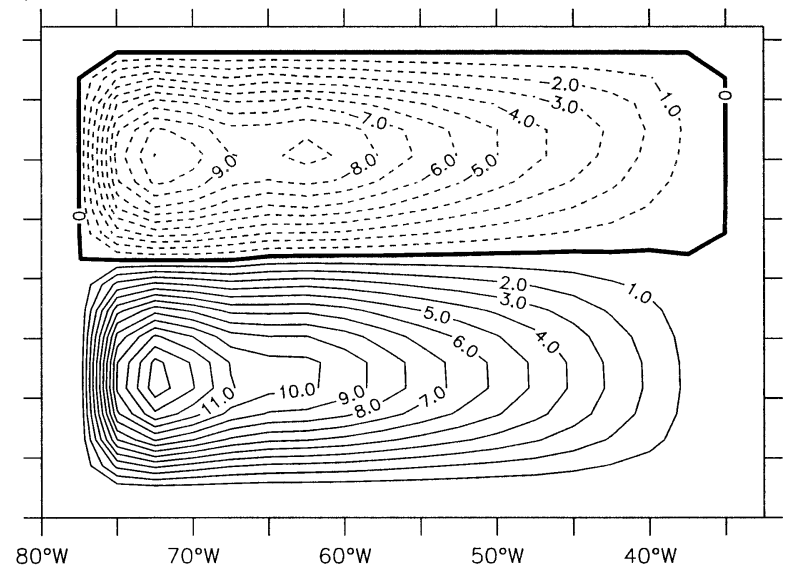

FIG. 6. Streamfunction (contour interval $1 \mathrm{~Sv}$ ) in experiment RIDGE in (a), (b) BARBI and (c), (d) MOM averaged over the (a), (c) first months of the integration and the (b), (d) 12th month.

shown). There is also good agreement between MOM and BARBI in the two density moments and in the baroclinic velocity moments. Figure 7 exemplifies the baroclinic velocity structure for MOM and BARBI after 4 years of integration. Shown are the vertical mean horizontal velocities in an upper layer of 2000-m depth and a deep layer reaching from $2000 \mathrm{~m}$ to the bottom. For BARBI we have projected the vertical structure of the vertical shear $\mathbf{u}^{\prime}$ on a linear profile $f_{2}=z+h / 2$ (see appendix A). At the early stages of the integration, the flow below $2000 \mathrm{~m}$ is comparable in magnitude with the upper layer flow (not shown). When the baroclinic adjustment is completed, as shown in Fig. 7, the flow in the lower layer becomes much weaker than in the upper layer. The upper-layer flow gets more and more shielded from the topography and the streamfunctions resembles the flat bottom Sverdrup solution.

The ridge experiment follows the scenario of the "Sverdrup catastrophy," as outlined in the work of Anderson and Gill (1975) for a vertically continuous model with flat bottom and Anderson and Killworth (1977) for a two-layer system with topography present. In the vertically integrated framework used in BARBI, the catas- trophy utters itself in a partial compensation between the Jacobian $\boldsymbol{\nabla} \psi \cdot \nabla(f / h)$ and the baroclinic bottom torque. Evaluating the steady state transport from $f \mathbf{k} \times$ $\mathbf{U}=-h \boldsymbol{\nabla} P-\boldsymbol{\nabla} E_{1}+\boldsymbol{\tau}_{S}+\mathbf{R}$ (where $\mathbf{R}$ denotes the lateral friction term), the Jacobian becomes

$$
\begin{aligned}
\underline{\nabla} \psi \cdot \nabla \frac{f}{h}= & \frac{\beta}{h} \frac{\partial \psi}{\partial x}-\frac{f}{h^{2}} \mathbf{U} \cdot \boldsymbol{\nabla} h \\
= & \frac{\beta}{h} \frac{\partial \psi}{\partial x}-\frac{1}{h} \underline{\nabla} P \cdot \boldsymbol{\nabla} h-\frac{1}{h^{2}} \underline{\nabla} E_{1} \cdot \boldsymbol{\nabla} h \\
& -\frac{1}{h^{2}}\left(\boldsymbol{\tau}_{S}+\mathbf{R}\right) \cdot \underline{\nabla} h .
\end{aligned}
$$

The wave response of the Sverdrup catastrophy cancels out the deep pressure gradient, $\left.(\nabla p)\right|_{-h}=\nabla P-$ $g \rho(-h) \nabla h=0$, and thus $\boldsymbol{\nabla} P \| \nabla h$, which switches off the Jacobian of $P$ and $h$ in Eq. (12). The compensation of the bottom pressure gradient and the corresponding cancellation of the bottom pressure torque $\underline{\nabla} P \cdot \nabla h$ can be traced back to the suggestions by Neumann (1955) and Welander (1959). Notice that this does lead to the cancellation of the JEBAR torque $\boldsymbol{\nabla} E_{1} \cdot \nabla h$. The rela- 

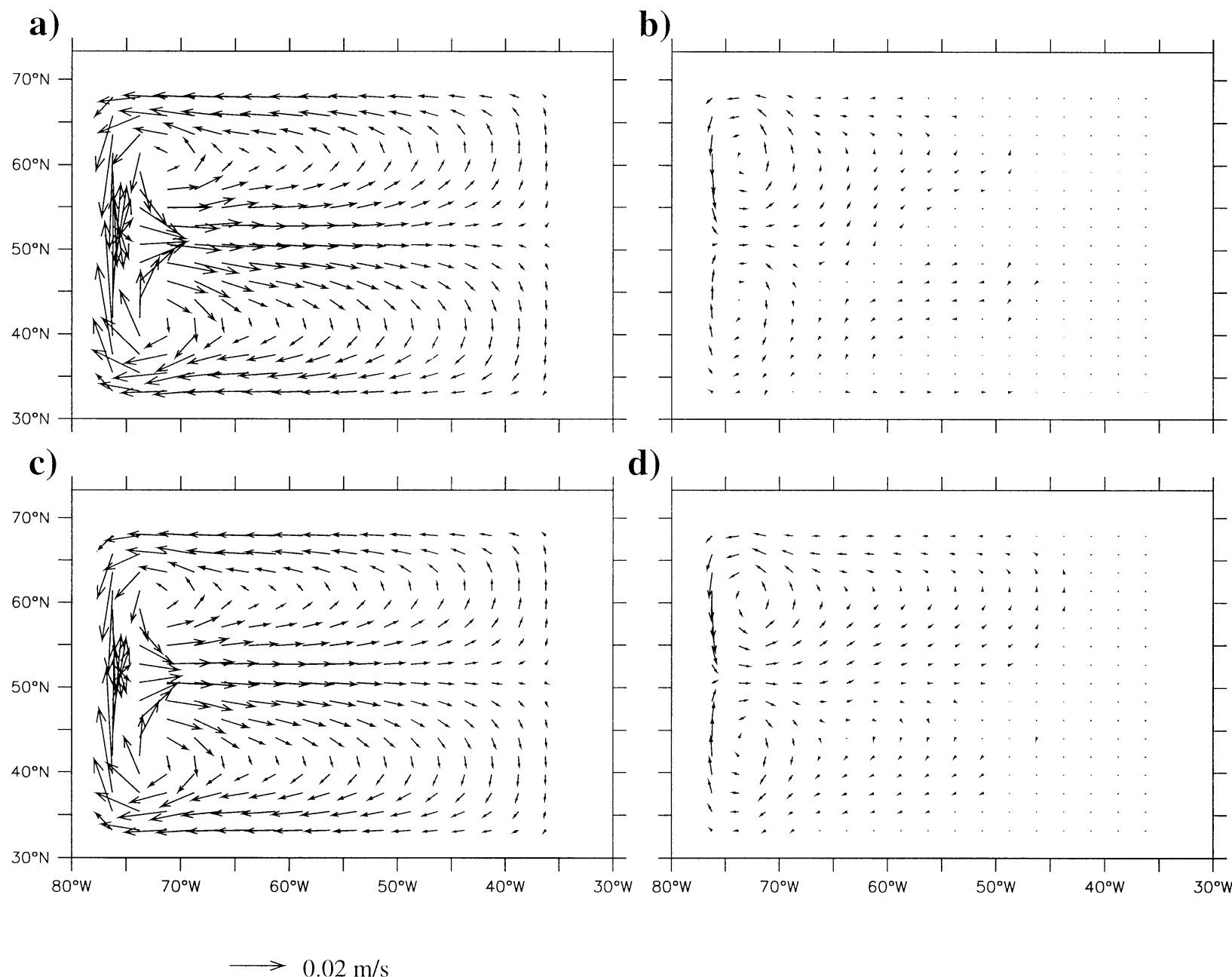

d)

FIG. 7. (a), (c) Upper- and (b), (d) lower-layer velocity in experiment RIDGE in (a), (b) BARBI and (c), (d) MOM at the end of the fourth year of the integrations. The upper-layer depth is chosen as $2000 \mathrm{~m}$. Also see appendix A for definitions of layer velocities in BARBI.

tion Eq. (12) then implies that the baroclinic bottom torque cancels in the vorticity equation Eq. (2), the forcing turns to $\left(\underline{\boldsymbol{\nabla}} \cdot \boldsymbol{\tau}_{S}\right) / h$, and the $f / h$ characteristics are replaced by $f$ characteristics: that is, the vorticity balance is that of flat-bottom conditions except for the frictional terms.

\section{d. Experiment ATLACC}

The experiment ATLACC is a test of the performance of BARBI in a more realistic application. The domain is the entire Atlantic and the region around Antarctica. The North Pole of the model grid is rotated to a point at the equator in the Pacific. The horizontal resolution is $2^{\circ}$ and BARBI uses truncation at order 3 , with $E_{3}=$ $0.3921 h^{2} E_{1}$. The horizontal viscosity is $2 \times 10^{4} \mathrm{~m}^{2} \mathrm{~s}^{-1}$, other parameters are the same as in DISTURB. The model is forced with interpolated annual mean wind stress data from an European Centre for Medium-Range Weather Forecasts (ECMWF) analysis (Barnier et al.
1995) (note that we omit surface density forcing for the moment). The topography was interpolated from the ETOPO5 (National Geophysical Data Center 1988) dataset onto the model grid and smoothed with a twodimensional symmetric filter. Figure 8 shows the topography and wind stress used in ATLACC.

We present results of BARBI from three different experiments using the ATLACC setup: an experiment without background stratification $\left(N_{0} \equiv 0\right.$, notice that without thermohaline forcing the density moments become zero, thus a case without background stratification is strictly barotropic) but with topography, an experiment with a flat bottom $(h=5500 \mathrm{~m})$, and an experiment with the interpolated topography and background stratification $\left(N_{0}=2.6 \times 10^{-3} \mathrm{~s}^{-1}\right)$. This suite of three experiments follows the, by now classical, simulations with the early GFDL (MOM) model, described by Bryan and Cox (1972) and Cox (1972). Similar experiments are found in Han (1984a,b) and Cai and Baines (1996) 
a)

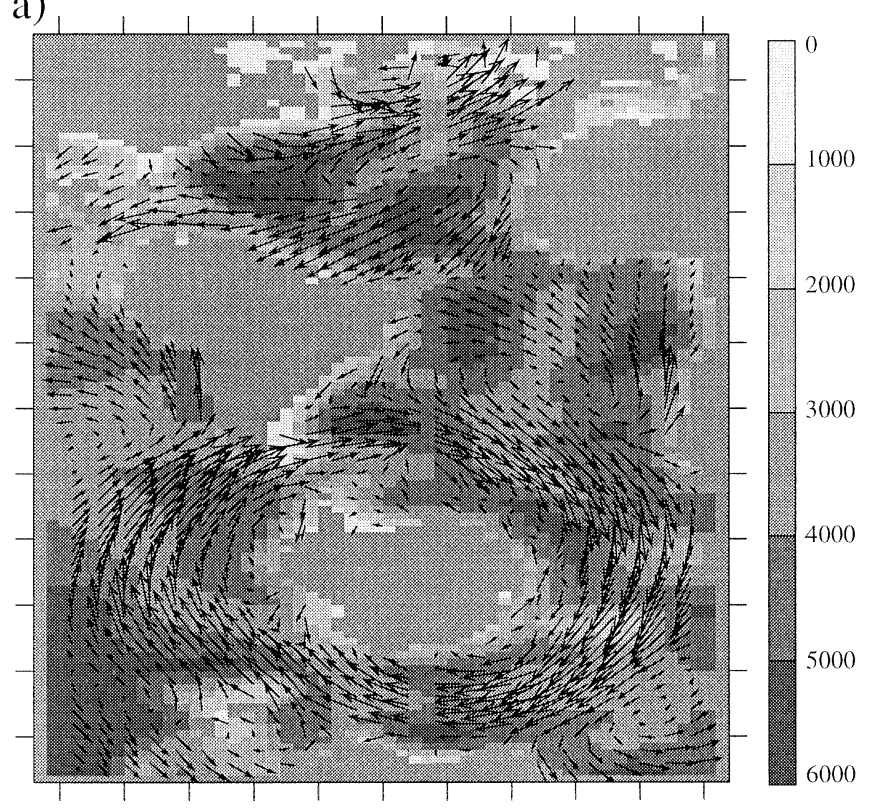

b)

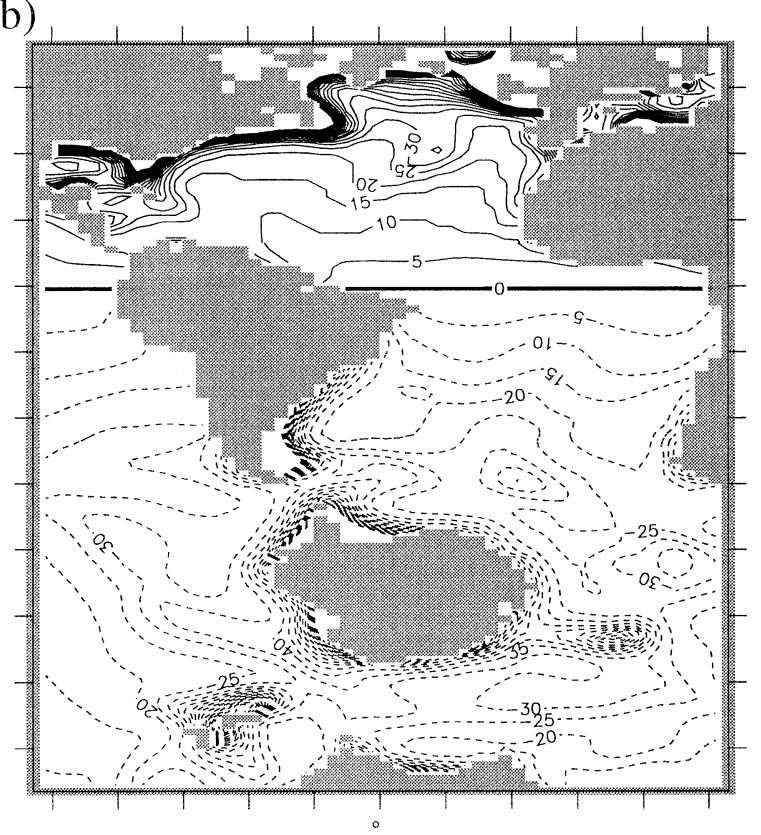

FIG. 8. (a) Topography and wind stress in experiment ATLACC. (b) The $f / h$ contours in experiment ATLACC.

for the global ocean, and in Olbers and Wübber (1991) for a circumpolar domain.

Here, we want to briefly summarize the most important results of these studies concerning the Antarctic Circumpolar Current (ACC) since we did not repeat similar experiments with MOM ourselves. The transport of the ACC varies in the three experiment enormously. The homogeneous ocean with topography has very low ACC transport [22 Sv in Cox (1972)] since the geostrophic contours are blocked by the sill in Drake Passage, except for some contours on the shallow continental shelves (see Fig. 8). The transport along blocked contours is small: the sink for the momentum input by wind is form drag involving the pressure differences between the continents. In contrast, the flat-bottom, homogeneous ocean has several hundreds of Sverdrups (more than $600 \mathrm{~Sv}$ in Bryan and Cox 1972, reflecting "Hidaka's dilemma" Wolff et al. 1991). Here, friction is the only momentum sink, with the effect that either unrealistically large transports are obtained or unrealistically large eddy viscosities have to be considered (the flat-bottom transport is proportional to $\tau_{S} / A_{h}$ ). The third experiment, now considering baroclinic conditions in a topographic ocean, generally gets a realistic transport for the ACC [187 Sv in Cox (1972)]. The importance of blocked geostrophic contours for the strength of the ACC and the relief from this constraint in a baroclinic ocean were recently investigated by Borowski et al. (2002).

Figure 9 and 10 show the streamfunctions for the three BARBI experiments; for the baroclinic case, the density moment $E_{1}$ is shown as well. Before discussing the ACC, we just note in passing that the model behavior in the North Atlantic of the three configurations reveals the regimes discussed in the experiments RIDGE, that is, the shift from the topographic Sverdrup regime to the flat-bottom one, if baroclinicity is included. However, while the subtropical gyre with maximal transports of about $30 \mathrm{~Sv}$ for the baroclinic case with topography is within rough bounds of observational estimates, the subpolar North Atlantic shows only a weak cyclonic circulation present in all three experiments, at a maximum of $5 \mathrm{~Sv}$ in the flat-bottom case. This is in contrast to observational estimates that give higher values of about 30-50 Sv, pointing toward the importance of thermohaline forcing for the strength of the North Atlantic subpolar gyre (Böning et al. 1996; Greatbatch et al. 1991), which we have excluded in our experiments.

For the ACC transport, the cases without stratification yield 35 and $700 \mathrm{~Sv}$ with and without topography, respectively. Both circulations are quite unrealistic as discussed above. In the homogeneous topographic case the flow roughly follows $f / h$ contours, shown in Fig. 8b. Most $f / h$ contours are blocked and the flow passes Drake Passage in a narrow boundary layer near the South American shelf. There are a few domains around Antarctica with closed $f / h$ contours, leading to closed gyres, most prominently seen in the South Atlantic sector.

As expected, the ACC gets extremely vigorous in the flat-bottom case. The complete spinup in this experiment takes several decades, in contrast to applications in closed basins with flat bottom in which the spinup is completed after a couple of months at most. It is clear that the wave adjustment of the Sverdrup circulation (Anderson and Gill 1975) does not apply here since the 
a)

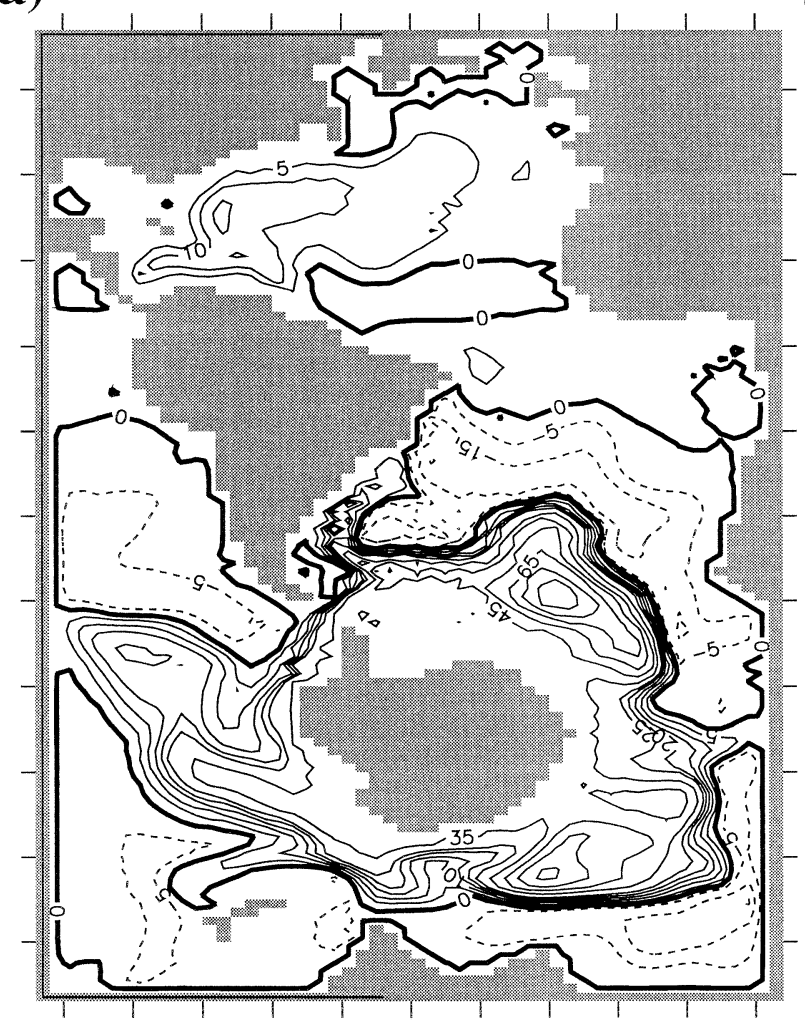

b)

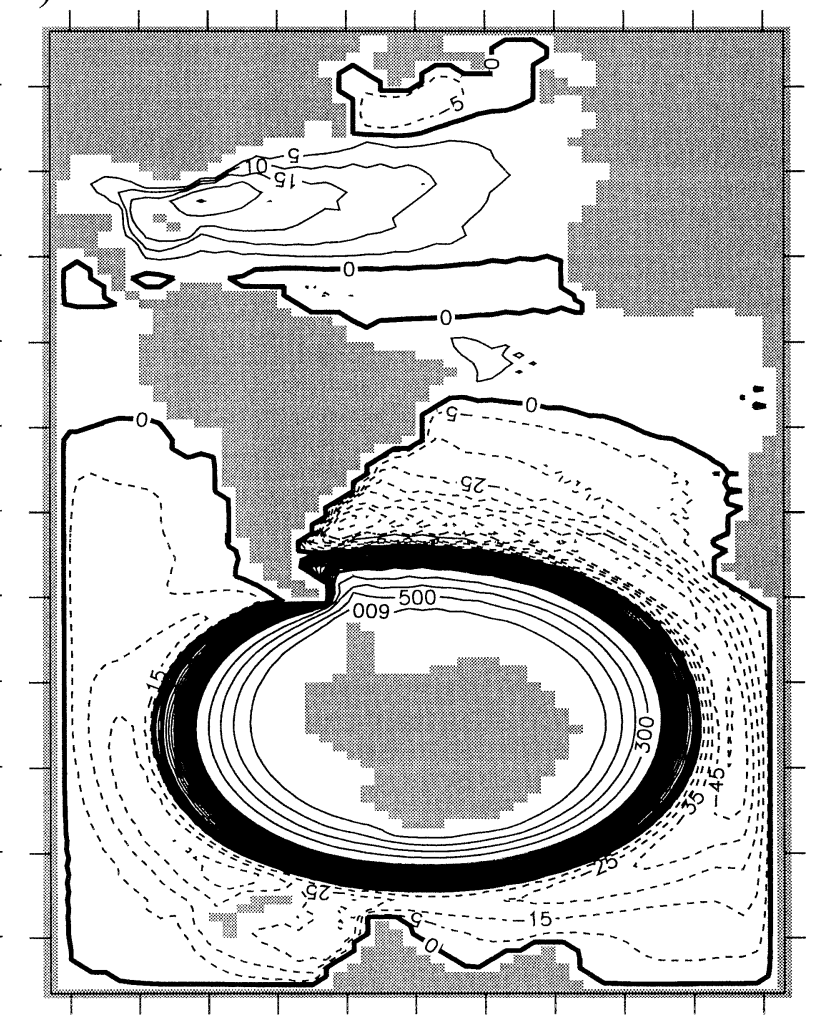

FIG. 9. (a) Streamfunction in experiment ATLACC with $N^{2}=0$ after 40 days of integration. (b) Same but here using a flat bottom instead of the realistic topography and after $1 \mathrm{yr}$ of integration. Contour intervals are $10 \mathrm{~Sv}$ for values lower than $-5 \mathrm{~Sv}, 5 \mathrm{~Sv}$ between -5 and $25 \mathrm{~Sv}, 10 \mathrm{~Sv}$ between 25 and $200 \mathrm{~Sv}$, and $100 \mathrm{~Sv}$ for values greater than $200 \mathrm{~Sv}$.

ACC system is supercritical (the eastward current overtakes the westward wave propagation).

With topography and stratification implemented, the flow around Antarctica becomes reasonable, with an ACC transport of about $130 \mathrm{~Sv}$ through Drake Passage [the good agreement with the observed value of $134 \pm$ $13 \mathrm{~Sv}$ should be viewed critically; for a recent review on the ACC see Rintoul et al. (2001)]. After passing Drake Passage, the ACC flows northward along the east coast of South America and detaches at about $40^{\circ} \mathrm{S}$ in a confluence with the Brazil Current. All basin gyres get a more realistic structure. The closed $f / h$ gyres around Antarctica have disappeared; now there are indications of subpolar gyres, for example, a closed circulation in the Weddell Sea with a strength of about 20 Sv.

In conclusion, the experiments in this more realistic setup confirm that a low-order BARBI model produces the important aspects of the wind-driven large-scale baroclinic circulation over topography.

\section{Concluding discussion}

\section{a. Summary}

We have described the physical ingredients and performance of a new type of ocean general circulation model with simplified physics, which we call BARBI. Motivation for the new approach comes from the appearance of the first vertical density moment (or vertically integrated potential energy) in the JEBAR torque of the vorticity balance of the barotropic transport. JEBAR is the predominant coupling of the large-scale, barotropic circulation to the baroclinic modes in the vorticity balance and resembles, together with the wind stress curl, the forcing of the barotropic flow.

Using the first density moment in JEBAR as a guideline, we have projected the primitive equations on vertical powers of depth. As a consequence of the projection, all variables in BARBI become two-dimensional since the vertical coordinate was transformed into the order of the vertical moments of the baroclinic variables. Projection of the thermohaline balance yields prognostic equations for vertical density moments $E_{n}$, such that we are able to calculate the complete budget of barotropic vorticity and thus the barotropic transport. One important effect on $E_{n}$, lifting of the background stratification by the baroclinic flow, leads, in a natural way, to the definition of "baroclinic velocity moments," which are thus added to the coupled hierarchy of baroclinic variables in BARBI to close the model.

We have derived linear solutions of the model equations and show that the entire spectrum of oceanic waves 
a)

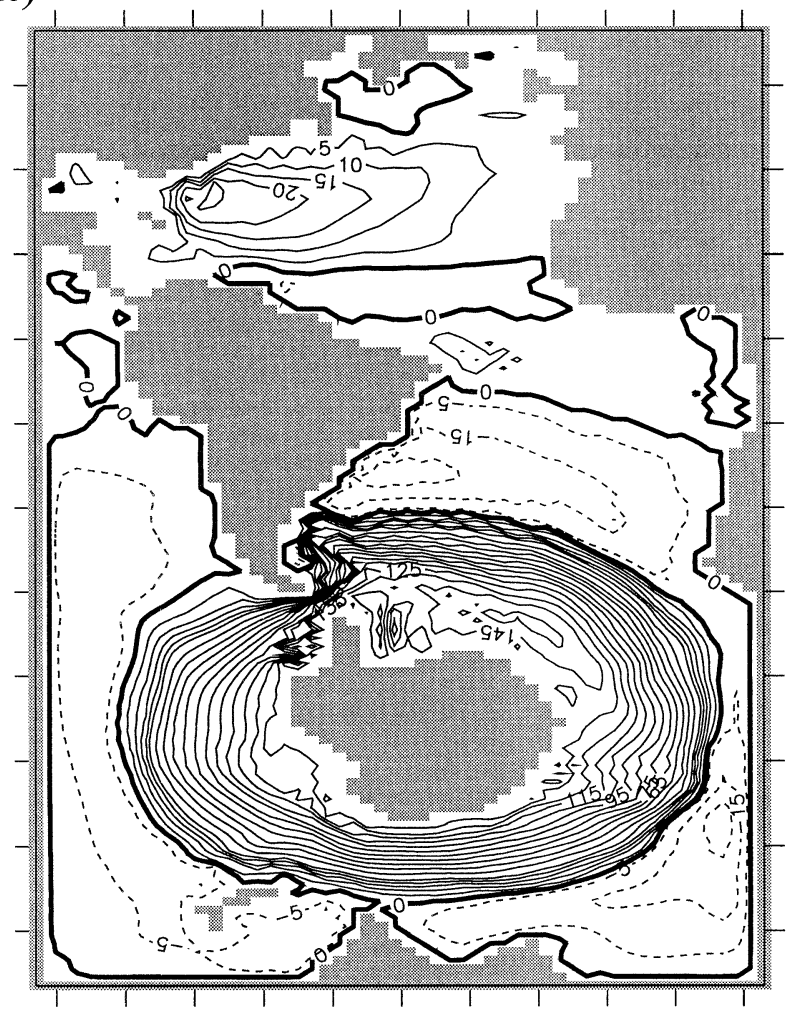

b)

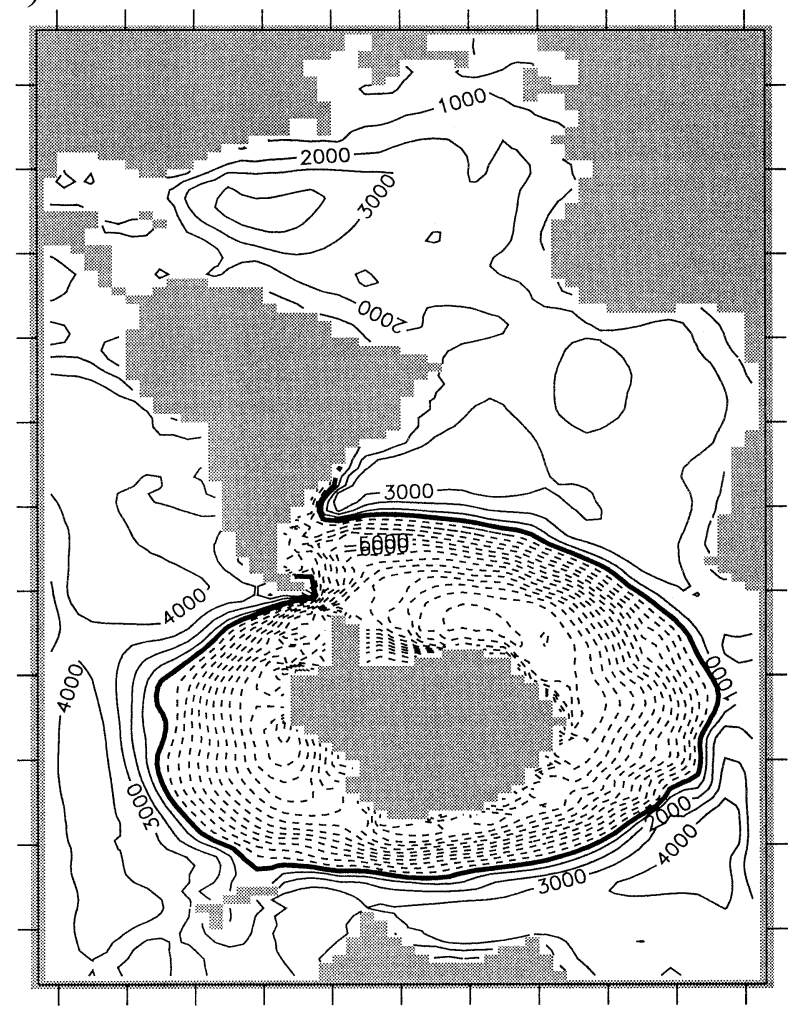

FIG. 10. (a) Streamfunction in experiment ATLACC with $N^{2}=2.6 \times 10^{-3} \mathrm{~s}^{-1}$ and realistic topography after 40 days of integration. Contour intervals are $10 \mathrm{~Sv}$ for values lower than $-5 \mathrm{~Sv}, 5 \mathrm{~Sv}$ between -5 and $25 \mathrm{~Sv}$, and $10 \mathrm{~Sv}$ for values greater than $25 \mathrm{~Sv}$. (b) The first density moment $E_{1}$ with a contour interval of $1000 \mathrm{~m}^{2} \mathrm{~s}^{-2}$ in the same experiment.

exists in BARBI. A practical truncation procedure is suggested, cutting off the density hierarchy at a certain number of moments and preserving the flat bottom and topographically modified wave spectrum exactly (for the resolved modes). We have demonstrated the ability of the truncated BARBI model to simulate some fundamental problems of wave variability and wind-driven, baroclinic circulation over topography in the ocean. In fact, in these experiments BARBI, consisting of only a few two-dimensional equations, compares very well with a full primitive equation level model with high vertical resolution.

Last, we want to note that BARBI is open for testing (the source code could be downloaded online at http:// www.ifm.uni-kiel.de/fb/fb1/tm/data/pers/ceden/BARBI/ BARBI.html) with the restrictions on the physics reported below.

\section{b. Discussion}

In our view, the most important advantage of this new model concept, as compared with other models with simplified physics, is that the fundamental role of the effect of topography and baroclinicity in generating large-scale ocean transports is correctly represented in the model equations without any truncation. So far, the correct representation of topography in simple ocean models has not received much attention, but is, however, an important factor controlling the barotropic and baroclinic flow. Therefore, we believe that BARBI is likely to contribute to a better understanding of the large-scale ocean dynamics, both by analytical considerations and results from numerical integrations.

Another advantage in comparison with, for example, a quasigeostrophic model or a reduced-gravity model, is that it is in principle possible to include the effect of surface buoyancy forcing. We have not explored this feature in the present manuscript since smallness of the perturbation density was assumed, which will not be the case for strong thermohaline forcing (but, however, is valid for the wave and wind-driven cases in the present study). Building a simplified, two-dimensional model for the wind and thermohaline-driven, large-scale flow will be the aim of a companion study in preparation.

A drawback of the truncated BARBI model is that it is not possible to derive exact and closed forms (in terms of the modeled $E_{n}$ and $\mathbf{U}_{n+1}^{\prime}$ ) of the effects by the baroclinic advection of perturbation density and, for nonconstant $N$, by the baroclinic lifting of the background stratification. The treatment of these effects on the resolved $E_{n}$ in a truncated BARBI model involves vertical moments of higher order as the order of truncation. On 
the other hand, it is straightforward to use the parameterization of the higher order truncated $E_{n}$, as used in the truncation procedure, to evaluate both effects, as outlined in section 4. Note that utilizing an expansion of the vertical coordinate in the basis of modified Legendre polynomials, it is in principle possible to relax almost all the remaining assumptions that we make in the present study concerning dissipative and diabatic terms and neglection of momentum advection.

We want to stress that this is the only point in which BARBI comes close to a normal spectral model, to the respect that the order of truncation affects nonlinear terms in the lower-order model equations. However, in BARBI, the truncation procedure offers an efficient and elegant way to parameterize such effects, instead of simply neglecting them, as is normally done in spectral models. Furthermore and in contrast to spectral models (and, e.g., layered ocean models), our choice of the structure functions is motivated for physical reasons, based on the underlying primitive equations itself by the appearance of the vertically integrated potential energy in the bottom torque of the barotropic vorticity, resembling the fundamental agent for interaction of the large-scale, barotropic, and baroclinic flow. Describing this interaction is, in turn, the major task for BARBI.

Acknowledgments. We thank Richard Greatbatch for valuable comments. Dirk Olbers is grateful for support from Richard Greatbatch's NSERC research grant during a visit to Halifax in September 2001. Comments by two anonymous reviewers and the editor helped to improve this manuscript.

\section{APPENDIX A}

\section{Evaluation of $S_{n}^{\prime \prime}$}

An exact integration of $S_{n}^{\prime \prime}$ down to BARBI's baroclinic state variables (the density and velocity moments) is not possible. However, we can assume an approximative representation of the vertical structure of either the baroclinic velocity or the density perturbation. For the purpose of demonstration we proceed by representing $\mathbf{u}^{\prime}$ in terms of polynomials $f_{j}(z, h)$ of the vertical coordinate $z$,

$$
\mathbf{u}^{\prime}(\mathbf{x}, z, t)=\sum_{j=1}^{J} \mathbf{v}_{j}(\mathbf{x}, t) f_{j}(z, h) .
$$

It is reasonable to assume that the $f_{j}$ have zero integral (i.e., they should be orthonormal-this makes them dependent on $h$ ) so that each single term in Eq. (A1) integrates to zero. The coefficient vectors $\mathbf{v}_{j}$ can be expressed as a linear combination of the baroclinic velocity variables by inversion of $\mathbf{u}_{k}^{\prime}=\Sigma_{j} a_{k j} \mathbf{v}_{j}$ where the matrix $a_{k j}=\int z^{k} f_{j} d z$ is generated by projection of Eq. (A1) onto powers of $z$. The vertical baroclinic velocity then becomes

$$
\begin{aligned}
w^{\prime} & =-\boldsymbol{\nabla} \cdot \sum_{j=1}^{J} \mathbf{v}_{j}(\mathbf{x}, t) \int_{-h}^{z} f_{j}(z, h) d z \\
& =-\sum_{j=1}^{J} \boldsymbol{\nabla} \cdot \mathbf{v}_{j}(\mathbf{x}, t) g_{j}(z, h),
\end{aligned}
$$

where $g_{j}(z, h)$ is the integral of $f_{j}$ appearing in the first expression above. Inserting these expressions into Eq. (5) one finds

$$
\begin{aligned}
S_{n}^{\prime \prime}=-g \sum_{j}\left\{\boldsymbol{\nabla} \cdot\left[\mathbf{v}_{j} \int_{-h}^{0} \rho z^{n} f_{j}(z, h) d z\right]\right. \\
\left.+n \int_{-h}^{0} \rho z^{n-1} \boldsymbol{\nabla} \cdot \mathbf{v}_{j} g_{j}(z, h) d z\right\} .
\end{aligned}
$$

It becomes obvious that the vertical integration generates a series of density moments $E_{k}$ of order $k \geq n$. Thus, $S_{n}^{\prime \prime}$ is entirely determined as a functional of BARBI's state variables $E_{k}$ and $\mathbf{u}_{j}^{\prime}$.

The choice of the $f_{j}$ is crucial as to which moments will appear (modified Legendre polynomials of depth are a reasonable choice). In particular, if odd powers of $z$ are in the $f_{j}$, then even moments of density will emerge in the source terms $S_{n}^{\prime \prime}$ with odd index. However, in general, odd and even moments will be involved, similar to the consequence of a nonconstant $N^{2}$ (density moments of higher index as $n+2$ will be involved as well). Obviously, the parameterization of higher order moments, being not part of the truncated model, should follow the procedure presented in section 4 for the case of a nonconstant $N^{2}$.

\section{APPENDIX B}

\section{Frictional and Diabatic Closures}

In this section, we consider the frictional sources in the barotropic and baroclinic momentum budgets Eq. (1) and Eq. (6) and the diabatic sources in the tendency equation (4) for $E_{n}$. We start with the frictional forces in the barotropic momentum balance and stick to the usual parameterization for the divergence of $\mathbf{J}_{u}$,

$$
\frac{\partial\left(\mathbf{J}_{u}\right)_{i j}}{\partial x_{j}}=\frac{\partial}{\partial x_{j}}\left(A^{h} \frac{\partial u_{i}}{\partial x_{j}}\right),
$$

where $A^{h}$ denotes a horizontal viscosity and $x_{j}, j=1$, 2 shorthand for $x, y$ (similar for $u_{j}$ ). The barotropic frictional force is then

$$
\begin{aligned}
\mathbf{F} & =\boldsymbol{\tau}_{S}-\boldsymbol{\tau}(-h)+\int_{-h}^{0} \frac{\partial}{\partial x_{j}}\left(A^{h} \frac{\partial \mathbf{u}}{\partial x_{j}}\right) d z \\
& =\boldsymbol{\tau}_{S}-\boldsymbol{\tau}(-h)+A^{h}\left(\frac{\partial \mathbf{u}}{\partial x_{j}}\right)_{-h} \frac{\partial h}{\partial x_{j}}+\frac{\partial}{\partial x_{j}} \int_{-h}^{0} A^{h} \frac{\partial \mathbf{u}}{\partial x_{j}} d z .
\end{aligned}
$$

Notice that the bottom stress $\boldsymbol{\tau}(-h)$ is not the only agent transferring barotropic momentum into the bottom. 
When the bottom is inclined, there is a nondivergent contribution from $\boldsymbol{\nabla} h \cdot \mathbf{J}_{u}(-h)$. However, we want to keep our parameterization simple and set the transfer of momentum into the bottom altogether to zero; that is, $-\boldsymbol{\tau}(-h)+A^{h}\left(\partial \mathbf{u} / \partial x_{j}\right)_{-h} \partial h / \partial x_{j}=0$ (free slip at the bottom). Moreover, we are using a constant lateral viscosity $A_{h}$. The remaining barotropic frictional force is then

$$
\mathbf{F}=\boldsymbol{\tau}_{s}+A^{h} \frac{\partial}{\partial x_{j}}\left[h \frac{\partial \mathbf{U} / h}{\partial x_{j}}+\mathbf{u}^{\prime}(-h) \frac{\partial h}{\partial x_{j}}\right] .
$$

With the above frictional parameterization there is coupling of the barotropic and the baroclinic motion. We tend to regard the effect of frictionally induced coupling as minor. In addition, this form of frictional coupling could be viewed as a result of our simple (but standard) parameterization of frictional fluxes. Therefore, we skip the term $\mathbf{u}^{\prime}(-h) \partial h / \partial x_{j}$, and the full barotropic momentum equation now reads

$$
\frac{\partial \mathbf{U}}{\partial t}+f \mathbf{k} \times \mathbf{U}=-h \boldsymbol{\nabla} P-\boldsymbol{\nabla} E_{1}+\boldsymbol{\tau}_{S}+A^{h} \frac{\partial}{\partial x_{j}} h \frac{\partial \mathbf{U} / h}{\partial x_{j}} .
$$

Note that there is no transfer of barotropic momentum into the bottom by frictional forces in this parameterization.

Next we specify the frictional forces in the baroclinic momentum balance. Using again the above standard parameterization of frictional fluxes with constant lateral $\left(A^{h}\right)$ and vertical $\left(A^{v}\right)$ viscosity gives

$$
\mathbf{F}^{\prime}=\left(1-\frac{1}{h} \int_{-h}^{0} d z\right)\left(A^{h} \nabla^{2} \mathbf{u}^{\prime}+A^{v} \frac{\partial^{2} \mathbf{u}^{\prime}}{\partial z^{2}}\right) .
$$

To be consistent we are using a free-slip condition at the bottom as for the barotropic momentum; that is, $A^{v} \partial \mathbf{u}^{\prime} / \partial z=\boldsymbol{\tau}_{S}$ at the sea surface and $A^{v} \partial \mathbf{u}^{\prime} / \partial z+A^{h}(\nabla h$ $\cdot \boldsymbol{\nabla}) \mathbf{u}^{\prime}=0$ at the bottom. Multiplying the frictional force in the baroclinic momentum balance with $z^{n}$ and integration over depth yields

$$
\begin{aligned}
\int_{-h}^{0} z^{n} \mathbf{F}^{\prime} d z= & (-1)^{n} \frac{h^{n+1}}{n+2} \boldsymbol{\tau}_{S}+A^{h} \nabla^{2} \mathbf{u}_{n+1}^{\prime} \\
& +A^{v}(n+1)\left[n \mathbf{u}_{n-1}^{\prime}+(-1)^{n} h^{n} \mathbf{u}^{\prime}(-h)\right] \\
& +A^{h} \phi_{n+1}^{\prime} .
\end{aligned}
$$

Note that the stress condition at the surface introduces the wind stress $\tau_{S}$. Many terms in this projection cancel out by the free-slip boundary condition at the bottom, but terms involving $\mathbf{u}^{\prime}(-h) \nabla^{2} h$ and $(\boldsymbol{\nabla} h \cdot \nabla) \mathbf{u}^{\prime}(-h)$ remain and are combined in $\boldsymbol{\phi}_{n+1}^{\prime}$. They are of order of the bottom slope times the magnitude of the friction terms retained. As a first step we ignore $\boldsymbol{\phi}_{n+1}^{\prime}$ entirely (because it is small) but also cancel the $\mathbf{u}^{\prime}(-h)$ term and write the baroclinic momentum balance as

$$
\begin{aligned}
\frac{\partial \mathbf{u}_{n+1}^{\prime}}{\partial t} & +f \mathbf{k} \times \mathbf{u}_{n+1}^{\prime} \\
= & -\frac{1}{n+2}\left[(-1)^{n} h^{n+1} \boldsymbol{\nabla} E_{1}+\boldsymbol{\nabla} E_{n+2}\right] \\
& +A^{v} n(n+1) \mathbf{u}_{n-1}^{\prime}+\frac{(-1)^{n} h^{n+1}}{n+2} \boldsymbol{\tau}_{S}+A^{h} \nabla^{2} \mathbf{u}_{n+1}^{\prime} .
\end{aligned}
$$

With this frictional closure each moment $\mathbf{u}_{n+1}^{\prime}$ is determined from a finite number of equations involving only lower $\mathbf{u}^{\prime}$ moments.

Last, we need a closure for the diabatic sources $D_{n}$ and $Q_{n}$ in the tendency equation for $E_{n}$. With the usual diffusive parameterization ${ }^{\mathrm{B} 1}$ of the turbulent density transport $\mathbf{J}_{\rho}=-K^{h} \boldsymbol{\nabla} \rho$, the $D_{n}$ term is evaluated as

$$
\begin{aligned}
D_{n} & =g \boldsymbol{\nabla} \cdot \int_{-h}^{0} z^{n} K^{h} \boldsymbol{\nabla} \rho d z \\
& =K^{h} \boldsymbol{\nabla} \cdot\left[\boldsymbol{\nabla} E_{n}+(-1)^{n+1} g h^{n} \rho(-h) \boldsymbol{\nabla} h\right] .
\end{aligned}
$$

This form points out that the density moments do not diffuse downgradient. As a first step, however, we ignore corrections involving $\nabla h$ and replace $D_{n}$ simply by

$$
D_{n}=K^{h} \nabla^{2} E_{n} \text {. }
$$

The vertical turbulent flux $B_{\rho}$ has a diffusive and convective property, and thus it should be a destruction of stratification and thus of total potential energy. On the other hand, $B_{\rho}$ also incorporates the flux of buoyancy from the surface, which could be a source or sink of potential energy. Parameterizations of these processes and appropriate forms of the $Q_{n}$ will be worked out in a companion part of the article. In the wave and winddriven experiments discussed in this study we have $Q_{n}$ $=0$.

\section{APPENDIX C}

\section{Flat-Bottom and Topographic Waves in the Infinite Hierarchy}

BARBI has the correct phase speeds for all baroclinic modes in the limit of infinite number of density moments, both for gravity and Rossby waves. To prove this, consider the eigenvalue problem Eq. (11), which in untruncated form is an infinite recurrence relation,

$$
\frac{1}{2 l(2 l+1)}\left(e_{2 l+1}-e_{1}\right)=\zeta e_{2 l-1}
$$

with eigenvalue $\zeta$, which, for instance, for Rossby waves

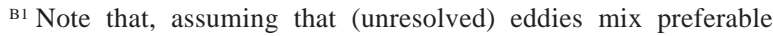
along isopycnals, this flux would go to zero for a linear equation of state. However, we keep this contribution, since we are aware that our numerical implementation of BARBI will need some kind of lateral diffusion of $E_{n}$. We suppress as well any attempt to include advective effects of eddy activity (bolus transport).
} 
is $\zeta=\omega /\left(\beta \kappa_{1} N_{0}^{2} h^{2} / f^{2}\right.$ ) (see section 4). It can be solved exactly by repeated substitution, which yields

$$
\begin{aligned}
& e_{1}\left[\frac{1}{(2 l+1) !}+\frac{\zeta}{(2 l-1) !}+\cdots+\frac{\zeta^{l-1}}{3 !}+\zeta^{l}\right] \\
& \quad=\frac{e_{2 l+1}}{(2 l+1) !} \text { or } \\
& e_{1}\left[1+\frac{1}{3 ! \zeta}+\frac{1}{5 ! \zeta^{2}}+\cdots+\frac{1}{(2 l+1) ! \zeta^{l}}\right] \\
& \quad=\frac{e_{2 l+1}}{(2 l+1) ! \zeta^{l}} .
\end{aligned}
$$

In the limit $l \rightarrow \infty$ the terms in the bracket tend to $\sqrt{-\zeta} \sin (1 / \sqrt{-\zeta})$ and, since the $e_{l}$ must remain finite, we arrive at the familiar sinusoidal constraint $\sin 1 /$ $\sqrt{-\zeta}=0$, and thus the eigenvalues $\zeta_{\nu}=-1 /(\nu \pi)^{2}, \nu$ $=1,2, \ldots$ of the baroclinic modes for constant $N$ are gained.

When topography is present, the wave properties get considerably more complicated because the barotropic and baroclinic variables get coupled. Here, we give a brief summary of what we found concerning the influence of topography on waves in BARBI. However, we restrict ourselves to the geostrophic wave branch. BARBI wave properties with consideration of topography follow then from

$$
\begin{array}{r}
\frac{\partial}{\partial t} \nabla^{2} \psi+h \underline{\boldsymbol{\nabla}} \psi \cdot \boldsymbol{\nabla} \frac{f}{h}+\frac{1}{h} \underline{\nabla} G_{1} \cdot \boldsymbol{\nabla} h=0 \\
\frac{\partial}{\partial t} G_{2 l-1}+\frac{\beta \lambda^{2}}{2 l(2 l+1)} \frac{\partial}{\partial x}\left(G_{2 l+1}-G_{1}\right) \\
-\frac{f \lambda^{2}}{h(2 l+1)}\left(\underline{\nabla} G_{1} \cdot \boldsymbol{\nabla} h-f \underline{\nabla} \psi \cdot \nabla h\right)=0,
\end{array}
$$

where $\lambda=N_{0} h /|f|$ is the bulk baroclinic Rossby radius and $G_{n}=E_{n} / h^{n-1}$. A wave solution with amplitudes $e_{n}$ and $\Psi$, assuming constant $\boldsymbol{\alpha}=\boldsymbol{\nabla} h$ and $\boldsymbol{\beta}=\boldsymbol{\nabla}(f / h)$ and constant $h$ and $f$ where not differentiated, yields the eigenvalue problem

$$
\begin{aligned}
& -\left(\omega-\omega_{R}\right) f \Psi-\frac{\alpha_{B}}{p^{2}} e_{1}=0 \\
& -\omega e_{2 l-1}-\frac{\omega_{C}}{2 l(2 l+1)}\left(e_{2 l+1}-e_{1}\right) \\
& +\frac{\alpha_{B} \lambda^{2}}{2 l+1}\left(e_{1}-f \Psi\right)=0
\end{aligned}
$$

with the abbreviations (using the shorthand $\boldsymbol{\kappa} \times \boldsymbol{\alpha}=$ $\left.\kappa_{2} \alpha_{1}-\kappa_{1} \alpha_{2}\right) \omega_{R}=h \boldsymbol{\kappa} \times \boldsymbol{\beta} / p^{2}$, the frequency of a barotropic topographic-planetary wave, $\omega_{C}=-\beta \lambda^{2} \kappa_{1}$, the scale of flat-bottom baroclinic planetary waves and $\alpha_{B}=-f / h \boldsymbol{\kappa} \times \boldsymbol{\alpha}$. Notice that $\alpha_{B} / \kappa^{2}$ is the frequency of a topographic wave on an $f$ plane. The hierarchy may be cast into a recurrence relation for the $e_{n}$, which yields by iteration, in the limit $l \rightarrow \infty$, the following dispersion relation

$$
\tan m=-m \eta \frac{(|\boldsymbol{\kappa}| \lambda)^{2}-m^{2}}{(1-\eta)(|\boldsymbol{\kappa}| \lambda)^{2}-m^{2}}
$$

with $\omega=\omega_{C} / m^{2}$ and $\eta=\lambda^{2} \alpha_{B} / \omega_{C}=(f / h)\left(\kappa_{2} \alpha_{1} / \kappa_{1}-\right.$ $\left.\alpha_{2}\right) / \beta$. The flat-bottom case is recovered for $\boldsymbol{\alpha}=0$ or $\eta=0$.

In the long-wave limit (we have filtered short Rossby waves), Eq. (C1) is consistent with what is known about topographic waves in a stratified ocean (Rhines 1977; Killworth and Blundell 2003). For $(|\boldsymbol{\kappa}| \lambda)^{2} \ll 1$ we get the condition $\tan m=-m \eta$, the slow baroclinic waves in Rhines' terminology. The smallest solution from Eq. (C1) yields $\mathrm{m}^{2}=(|\boldsymbol{\kappa}| \lambda)^{2} /(1+\eta)$, the fast barotropic wave with dispersion relation $\omega=\omega_{R}$. The bottomtrapped (fast baroclinic) mode is contained in BARBI as well [take $m^{2}=-\mu^{2}$ with $\mu>0$ in Eq. (C1)]. Note that the topographic waves are considered here for general $\boldsymbol{\alpha}$, in contrast to Rhines (1977) (where $\alpha_{1}=0$ is assumed).

\section{REFERENCES}

Anderson, D. L. T., and A. E. Gill, 1975: Spin-up of a stratified ocean, with applications to upwelling. Deep-Sea Res., 22, 583-586.

_ , and P. D. Killworth, 1977: Spin-up of a stratified ocean, with topography. Deep-Sea Res., 24, 709-732.

Barnier, B., L. Siefridt, and P. Marchesiello, 1995: Thermal forcing for a global ocean circulation model using a three year climatology of ECMWF analysis. J. Mar. Syst., 6, 363-380.

Bleck, R., C. Rooth, D. Hu, and L. T. Smith, 1992: Salinity-driven thermocline transients in a wind- and thermohaline forced isopycnic coordinate model of the North Atlantic. J. Phys. Oceanogr., 22, 1486-1505.

Böning, C. W., F. O. Bryan, W. R. Holland, and R. Doescher, 1996: Deep-water formation and meridional overturning in a high-resolution model of the North Atlantic. J. Phys. Oceanogr., 26, $1142-1164$.

Borowski, D., R. Gerdes, and D. Olbers, 2002: Thermohaline and wind forcing of a circumpolar channel with blocked geostrophic contours. J. Phys. Oceanogr., 32, 2520-2540.

Bryan, K., and M. D. Cox, 1972: The circulation of the world ocean: A numerical study. Part I, A homogenous model. J. Phys. Oceanogr., 2, 319-335.

Cai, W., and P. Baines, 1996: Interactions between thermohaline- and wind-driven circulations and their relevance to the dynamics of the Antarctic Circumpolar Current, in a coarse-resolution global ocean general circulation model. J. Geophys. Res., 101, $14073-$ 14093.

Cox, M. D., 1972: A baroclinic numerical model of the world ocean: Preliminary results. Numerical Models of Ocean Circulation, R. O. Reid et al., Eds., National Academy of Sciences, 107-120.

Davey, M. K., W. W. Hsieh, and R. C. Wajsowicz, 1983: The free Kelvin wave with lateral and vertical viscosity. J. Phys. Oceanogr., 13, 2182-2191.

Greatbatch, R. J., A. F. Fanning, A. D. Goulding, and S. Levitus, 1991: A diagnosis of interpentadal circulation changes in the North Atlantic. J. Geophys. Res., 96, 22 009-22 023.

Han, Y.-J., 1984a: A numerical World Ocean general circulation model. Part I. Basic design and barotropic experiment. Dyn. Atmos. Oceans, 8, 107-140.

_ 1984 b: A numerical world ocean general circulation model. Part II. A baroclinic experiment. Dyn. Atmos. Oceans, 8, 141172. 
Holland, W. R., 1973: Baroclinic and topographic influences on the transport in western boundary currents. Geophys. Fluid Dyn., 4, 187-210.

Hsieh, W. W., M. K. Davey, and R. C. Wajsowicz, 1983: The free Kelvin wave in finite-difference numerical models. J. Phys Oceanogr., 13, 1383-1397.

Killworth, P. D., 1985: A two level wind and buoyancy-driven thermocline model. J. Phys. Oceanogr., 15, 1414-1432.

- and J. R. Blundell, 2003: Long extratropical planetary wave propagation in the presence of slowly varying mean flow and bottom topography. Part I: The local problem. J. Phys. Oceanogr., 33, 784-801.

National Geophysical Data Center, 1988: Digital relief of the surface of the Earth (ETOP05). National Geophysical Data Center Data Annoucenment 88-MGG-02.

Neumann, G., 1955: On the dynamics of wind-driven currents. Meteorological Papers, Vol. 2, No. 4, New York University College of Engineering, $32 \mathrm{pp}$.

Olbers, D., and C. Wübber, 1991: The role of wind and buoyancy forcing of the Antarctic Circumpolar Current. Strategies for Future Climate Research, M. Latif, Ed., Max-Planck-Institut fur Meteorologie, 161-191.

Pacanowski, R. C., 1995: MOM 2 Documentation, User's Guide and Reference Manual. GFDL Ocean Group Tech. Report, 232 pp.

Redler, R., K. Ketelsen, J. Dengg, and C. W. Böning, 1998: A highresolution model for the circulation of the Atlantic Ocean. Proc.
Fourth European SGI/CRAY MPP Workshop, Garching, Germany, Insititutes for Plasma Physics, 95-108.

Rhines, P., 1977: The dynamics of unsteady currents. The Sea, E. D. Goldberg, Ed., Marine Modeling, Vol. 6, John Wiley and Sons, 189-318.

Rintoul, S. R., C. Hughes, and D. Olbers, 2001: The Antarctic Circumpolar Current system. Ocean Circulation and Climate, G. Siedier, J. Gould, and J. Church, Eds., Academic Press, 271302.

Sarkisyan, A. S., and V. F. Ivanov, 1971: Joint effect of baroclinicity and bottom relief as an important factor in the dynamics of sea currents. Izv. Acad. Sci. USSR Atmos. Oceanic Phys., 7, 173188.

Schulman, E. E., and P. P. Niiler, 1970: Topographic effects on the wind-driven ocean circulation. Geophys. Fluid Dyn., 1, 439_ 462.

Stevens, D. P., 1990: On open boundary conditions for three dimensional primitiv equation ocean circulation models. Geophys. Astrophys. Fluid Dyn., 51, 103-133.

Welander, P., 1959: On the vertically integrated mass transport in the oceans. The Atmosphere and the Sea in Motion, B. Bolin, Ed., The Rockefeller Institute Press, 95-101.

Wolff, J.-O., E. Maier-Reimer, and D. J. Olbers, 1991: Wind-driven flow over topography in a zonal $\beta$-plane channel: A quasi-geostrophic model of the antarctic circumpolar current. J. Phys. Oceanogr., 21, 236-264. 